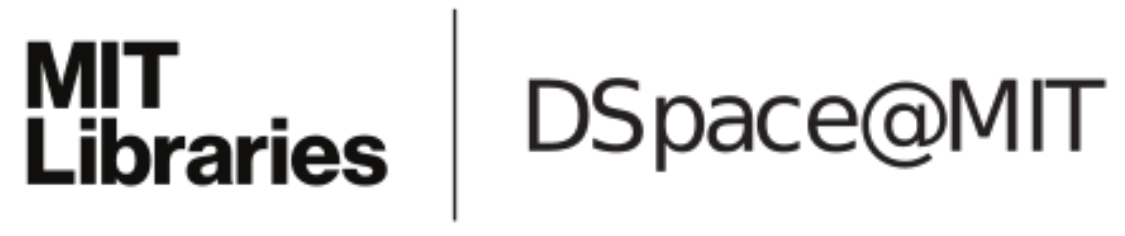

\author{
MIT Open Access Articles
}

Dolgopyat's method and the fractal uncertainty principle

The MIT Faculty has made this article openly available. Please share how this access benefits you. Your story matters.

Citation: Dyatlov, Semyon and Long Jin. "Dolgopyat's method and the fractal uncertainty principle." Analysis \& PDE 11, 6 (May 2018): 1457-1485 ( 2018 Mathematical Sciences Publishers

As Published: http://dx.doi.org/10.2140/APDE.2018.11.1457

Publisher: Mathematical Sciences Publishers

Persistent URL: https://hdl.handle.net/1721.1/123104

Version: Author's final manuscript: final author's manuscript post peer review, without publisher's formatting or copy editing

Terms of use: Creative Commons Attribution-Noncommercial-Share Alike 


\title{
DOLGOPYAT'S METHOD AND THE FRACTAL UNCERTAINTY PRINCIPLE
}

\author{
SEMYON DYATLOV AND LONG JIN
}

\begin{abstract}
We show a fractal uncertainty principle with exponent $\frac{1}{2}-\delta+\varepsilon, \varepsilon>0$, for Ahflors-David regular subsets of $\mathbb{R}$ of dimension $\delta \in(0,1)$. This improves over the volume bound $\frac{1}{2}-\delta$, and $\varepsilon$ is estimated explicitly in terms of the regularity constant of the set. The proof uses a version of techniques originating in the works of Dolgopyat, Naud, and Stoyanov on spectral radii of transfer operators. Here the group invariance of the set is replaced by its fractal structure. As an application, we quantify the result of Naud on spectral gaps for convex co-compact hyperbolic surfaces and obtain a new spectral gap for open quantum baker maps.
\end{abstract}

A fractal uncertainty principle (FUP) states that no function can be localized close to a fractal set in both position and frequency. Its most basic form is

$$
\left\|\mathbb{1}_{\Lambda(h)} \mathcal{F}_{h} \mathbb{1}_{\Lambda(h)}\right\|_{L^{2}(\mathbb{R}) \rightarrow L^{2}(\mathbb{R})}=\mathcal{O}\left(h^{\beta}\right) \quad \text { as } h \rightarrow 0
$$

where $\Lambda(h)$ is the $h$-neighborhood of a bounded set $\Lambda \subset \mathbb{R}, \beta$ is called the exponent of the uncertainty principle, and $\mathcal{F}_{h}$ is the semiclassical Fourier transform:

$$
\mathcal{F}_{h} u(\xi)=(2 \pi h)^{-1 / 2} \int_{\mathbb{R}} e^{-i x \xi / h} u(x) d x .
$$

We additionally assume that $\Lambda$ is an Ahlfors-David regular set (see Definition 1.1) of dimension $\delta \in(0,1)$ with some regularity constant $C_{R}>1$. Using the bounds $\left\|\mathcal{F}_{h}\right\|_{L^{2} \rightarrow L^{2}}=1,\left\|\mathcal{F}_{h}\right\|_{L^{1} \rightarrow L^{\infty}} \leq h^{-1 / 2}$, the Lebesgue volume bound $\mu_{L}(\Lambda(h)) \leq C h^{1-\delta}$, and Hölder's inequality, it is easy to obtain (1.1) with $\beta=\max \left(0, \frac{1}{2}-\delta\right)$.

Fractal uncertainty principles were applied by Dyatlov-Zahl [DyZa16], DyatlovJin [DyJi17], and Bourgain-Dyatlov [BoDy16] to the problem of essential spectral gap in quantum chaos: which open quantum chaotic systems have exponential decay of local energy at high frequency? A fractal uncertainty principle can be used to show local energy decay $\mathcal{O}\left(e^{-\beta t}\right)$, as was done for convex co-compact hyperbolic quotients in [DyZa16] and for open quantum baker's maps in [DyJi17]. Here $\Lambda$ is related to the set of all trapped classical trajectories of the system and (1.1) needs to be replaced by a more general statement, in particular allowing for a different phase in (1.2). The volume bound $\beta=\frac{1}{2}-\delta$ corresponds to the Patterson-Sullivan gap or more generally, the pressure gap. See $\S \S 4,5$ below for a more detailed discussion. 
A natural question is: can one obtain (1.1) with $\beta>\max \left(0, \frac{1}{2}-\delta\right)$, and if so, how does the size of the improvement depend on $\delta$ and $C_{R}$ ? Partial answers to this question have been obtained in the papers mentioned above:

- [DyZa16] obtained FUP with $\beta>0$ when $\left|\delta-\frac{1}{2}\right|$ is small depending on $C_{R}$, and gave the bound $\beta>\exp \left(-\mathbf{K}\left(1+\log ^{14} C_{R}\right)\right)$ where $\mathbf{K}$ is a global constant;

- [BoDy16] proved FUP with $\beta>0$ in the entire range $\delta \in(0,1)$, with no explicit bounds on the dependence of $\beta$ on $\delta, C_{R}$;

- [DyJi17] showed that discrete Cantor sets satisfy FUP with $\beta>\max \left(0, \frac{1}{2}-\delta\right)$ in the entire range $\delta \in(0,1)$ and obtained quantitative lower bounds on the size of the improvement - see $\S 5$ below.

Our main result, Theorem 1 , shows that FUP holds with $\beta>\frac{1}{2}-\delta$ in the case $\delta \in(0,1)$, and gives bounds on $\beta-\frac{1}{2}+\delta$ which are polynomial in $C_{R}$ and thus stronger than the ones in [DyZa16]. Applications include

- an essential spectral gap for convex co-compact hyperbolic surfaces of size $\beta>$ $\frac{1}{2}-\delta$, recovering and making quantitative the result of Naud [Na05], see $\S 4$;

- an essential spectral gap of size $\beta>\max \left(0, \frac{1}{2}-\delta\right)$ for open quantum baker's maps, extending the result of [DyJi17] to matrices whose sizes are not powers of the base, see $\S 5$. (For the case $\delta>\frac{1}{2}$ we use the results of [BoDy16] rather than Theorem 1.)

1.1. Statement of the result. We recall the following definition of Ahlfors-David regularity, which requires that a set (or a measure) has the same dimension $\delta$ at all points and on a range of scales:

Definition 1.1. Let $X \subset \mathbb{R}$ be compact, $\mu_{X}$ be a finite measure supported on $X$, and $\delta \in[0,1]$. We say that $\left(X, \mu_{X}\right)$ is $\delta$-regular up to scale $h \in[0,1)$ with regularity constant $C_{R} \geq 1$ if

- for each interval I of size $|I| \geq h$, we have $\mu_{X}(I) \leq C_{R}|I|^{\delta}$;

- if additionally $|I| \leq 1$ and the center of $I$ lies in $X$, then $\mu_{X}(I) \geq C_{R}^{-1}|I|^{\delta}$.

Our fractal uncertainty principle has a general form which allows for two different sets $X, Y$ of different dimensions in (1.1), replaces the Lebesgue measure by the fractal measures $\mu_{X}, \mu_{Y}$, and allows a general nondegenerate phase and amplitude in (1.2):

Theorem 1. Assume that $\left(X, \mu_{X}\right)$ is $\delta$-regular, and $\left(Y, \mu_{Y}\right)$ is $\delta^{\prime}$-regular, up to scale $h \in(0,1)$ with constant $C_{R}$, where $0<\delta, \delta^{\prime}<1$, and $X \subset I_{0}, Y \subset J_{0}$ for some intervals $I_{0}, J_{0}$. Consider an operator $\mathcal{B}_{h}: L^{1}\left(Y, \mu_{Y}\right) \rightarrow L^{\infty}\left(X, \mu_{X}\right)$ of the form

$$
\mathcal{B}_{h} f(x)=\int_{Y} \exp \left(\frac{i \Phi(x, y)}{h}\right) G(x, y) f(y) d \mu_{Y}(y)
$$

where $\Phi(x, y) \in C^{2}\left(I_{0} \times J_{0} ; \mathbb{R}\right)$ satisfies $\partial_{x y}^{2} \Phi \neq 0$ and $G(x, y) \in C^{1}\left(I_{0} \times J_{0} ; \mathbb{C}\right)$. 
Then there exist constants $C, \varepsilon_{0}>0$ such that

$$
\left\|\mathcal{B}_{h}\right\|_{L^{2}\left(Y, \mu_{Y}\right) \rightarrow L^{2}\left(X, \mu_{X}\right)} \leq C h^{\varepsilon_{0}} .
$$

Here $\varepsilon_{0}$ depends only on $\delta, \delta^{\prime}, C_{R}$ as follows

$$
\varepsilon_{0}=\left(5 C_{R}\right)^{-80\left(\frac{1}{\delta(1-\delta)}+\frac{1}{\delta^{\prime}\left(1-\delta^{\prime}\right)}\right)}
$$

and $C$ additionally depends on $I_{0}, J_{0}, \Phi, G$.

Remarks. 1. Theorem 1 implies the Lebesgue measure version of the FUP, (1.1), with exponent $\beta=\frac{1}{2}-\delta+\varepsilon_{0}$. Indeed, assume that $\left(\Lambda, \mu_{\Lambda}\right)$ is $\delta$-regular up to scale $h$ with constant $C_{R}$. Put $X:=\Lambda(h)$ and let $\mu_{X}$ be $h^{\delta-1}$ times the restriction of the Lebesgue measure to $X$. Then $\left(X, \mu_{X}\right)$ is $\delta$-regular up to scale $h$ with constant $30 C_{R}^{2}$, see Lemma 2.2. We apply Theorem 1 with $\left(Y, \mu_{Y}\right):=\left(X, \mu_{X}\right), G \equiv 1$, and $\Phi(x, y)=-x y$; then

$$
\left\|\mathbb{1}_{\Lambda(h)} \mathcal{F}_{h} \mathbb{1}_{\Lambda(h)}\right\|_{L^{2}(\mathbb{R}) \rightarrow L^{2}(\mathbb{R})}=\frac{h^{1 / 2-\delta}}{\sqrt{2 \pi}}\left\|\mathcal{B}_{h}\right\|_{L^{2}\left(X, \mu_{X}\right) \rightarrow L^{2}\left(X, \mu_{X}\right)} \leq C h^{1 / 2-\delta+\varepsilon_{0}} .
$$

2. Definition 1.1 is slightly stronger than [BoDy16, Definition 1.1] (where 'up to scale $h$ ' should be interpreted as 'on scales $h$ to 1 ') because it imposes an upper bound on $\mu_{L}(I)$ when $|I|>1$. However, this difference is insignificant as long as $X$ is compact. Indeed, if $X \subset[-R, R]$ for some integer $R>0$, then using upper bounds on $\mu_{L}$ on intervals of size 1 we get $\mu_{L}(I) \leq \mu_{L}(X) \leq 2 R C_{R} \leq 2 R C_{R}|I|^{\delta}$ for each interval $I$ of size $|I|>1$.

3. The restriction $\delta, \delta^{\prime}>0$ is essential. Indeed, if $\delta^{\prime}=0, Y=\{0\}, \mu_{Y}$ is the delta measure, and $f \equiv 1, G \equiv 1$, then $\left\|\mathcal{B}_{h} f\right\|_{L^{2}\left(X, \mu_{X}\right)}=\sqrt{\mu_{X}(X)}$. The restriction $\delta, \delta^{\prime}<1$ is technical, however in the application to Lebesgue measure FUP this restriction is not important since $\beta=\frac{1}{2}-\delta+\varepsilon_{0}<0$ when $\delta$ is close to 1 .

4. The constants in (1.5) are far from sharp. However, the dependence of $\varepsilon_{0}$ on $C_{R}$ cannot be removed entirely. Indeed, [DyJi17] gives examples of Cantor sets for which the best exponent $\varepsilon_{0}$ in (1.4) decays polynomially as $C_{R} \rightarrow \infty-$ see [DyJi17, Proposition 3.17] and $\S \S 5.2-5.3$.

1.2. Ideas of the proof. The proof of Theorem 1 is inspired by the method originally developed by Dolgopyat [Do98] and its application to essential spectral gaps for convex co-compact hyperbolic surfaces by Naud [Na05]. In fact, Theorem 1 implies a quantitative version of Naud's result, see $\S 4$. More recently, Dolgopyat's method has been applied to the spectral gap problem by Petkov-Stoyanov [PeSt10], Stoyanov [St11, St12], Oh-Winter [OhWi16], and Magee-Oh-Winter [MOW17].

We give a sketch of the proof, assuming for simplicity that $G \equiv 1$. For $f \in L^{2}\left(Y, \mu_{Y}\right)$, we have

$$
\left\|\mathcal{B}_{h} f\right\|_{L^{2}\left(X, \mu_{X}\right)} \leq \sqrt{\mu_{X}(X) \mu_{Y}(Y)} \cdot\|f\|_{L^{2}\left(Y, \mu_{Y}\right)},
$$


applying Hölder's inequality and the bound $\left\|\mathcal{B}_{h}\right\|_{L^{1}\left(X, \mu_{X}\right) \rightarrow L^{\infty}\left(Y, \mu_{Y}\right)} \leq 1$. However, under a mild assumption on the differences between the phases $\Phi(x, y)$ for different $x, y$, the resulting estimate is not sharp as illustrated by the following example where $X=Y=\{1,2\}, \mu_{X}(j)=\mu_{Y}(j)=\frac{1}{2}$ for $j=1,2$, and $\omega_{j \ell}:=\Phi(j, \ell) / h$ :

Lemma 1.2. Assume that $\omega_{j \ell} \in \mathbb{R}, j, \ell=1,2$, satisfy

$$
\tau:=\omega_{11}+\omega_{22}-\omega_{12}-\omega_{21} \notin 2 \pi \mathbb{Z}
$$

For $f_{1}, f_{2} \in \mathbb{C}$, put

$$
\left(\begin{array}{l}
u_{1} \\
u_{2}
\end{array}\right):=\frac{1}{2}\left(\begin{array}{ll}
\exp \left(i \omega_{11}\right) & \exp \left(i \omega_{12}\right) \\
\exp \left(i \omega_{21}\right) & \exp \left(i \omega_{22}\right)
\end{array}\right)\left(\begin{array}{l}
f_{1} \\
f_{2}
\end{array}\right) .
$$

Assume that $\left(f_{1}, f_{2}\right) \neq 0$. Then

$$
\left|u_{1}\right|^{2}+\left|u_{2}\right|^{2}<\left|f_{1}\right|^{2}+\left|f_{2}\right|^{2}
$$

Remark. Note that (1.8) cannot be replaced by either of the statements

$$
\left|u_{1}\right|+\left|u_{2}\right|<\left|f_{1}\right|+\left|f_{2}\right|, \quad \max \left(\left|u_{1}\right|,\left|u_{2}\right|\right)<\max \left(\left|f_{1}\right|,\left|f_{2}\right|\right) .
$$

Indeed, the first statement fails when $f_{1}=0, f_{2}=1$. The second one fails if $\omega_{11}=\omega_{12}$ and $f_{1}=f_{2}=1$. This explains why we use $L^{2}$ norms in the iteration step, Lemma 3.2.

Proof. We have

$$
\frac{\left|u_{1}\right|^{2}+\left|u_{2}\right|^{2}}{2} \leq \max \left(\left|u_{1}\right|^{2},\left|u_{2}\right|^{2}\right) \leq\left(\frac{\left|f_{1}\right|+\left|f_{2}\right|}{2}\right)^{2} \leq \frac{\left|f_{1}\right|^{2}+\left|f_{2}\right|^{2}}{2} .
$$

Assume that (1.8) does not hold. Then the inequalities in (1.9) have to be equalities, which implies that $\left|u_{1}\right|=\left|u_{2}\right|,\left|f_{1}\right|=\left|f_{2}\right|>0$, and for $a=1,2$

$$
\exp \left(i\left(\omega_{a 1}-\omega_{a 2}\right)\right) f_{1} \bar{f}_{2} \geq 0 \text {. }
$$

The latter statement contradicts (1.7).

To get the improvement $h^{\varepsilon_{0}}$ in (1.4), we use non-sharpness of (1.6) on many scales:

- We fix a large integer $L>1$ depending on $\delta, C_{R}$ and discretize $X$ and $Y$ on scales $1, L^{-1}, \ldots, L^{-K}$ where $h \sim L^{-K}$. This results in two trees of intervals $V_{X}, V_{Y}$, with vertices of height $k$ corresponding to intervals of length $\sim L^{-k}$.

- For each interval $J$ in the tree $V_{Y}$, we consider the function

$$
F_{J}(x)=\frac{1}{\mu_{Y}(J)} \exp \left(-\frac{i \Phi\left(x, y_{J}\right)}{h}\right) \mathcal{B}_{h}\left(\mathbb{1}_{J} f\right)(x),
$$

where $y_{J}$ is the center of $J$. The function $F_{J}$ oscillates on scale $h /|J|$. Thus both $F_{J}$ and the rescaled derivative $h|J|^{-1} F_{J}^{\prime}$ are controlled in uniform norm by $\|f\|_{L^{1}\left(Y, \mu_{Y}\right)}$. We express this fact using the spaces $\mathcal{C}_{\theta}$ introduced in $\S 2.2$. 
- If $J_{1}, \ldots, J_{B} \in V_{Y}$ are the children of $J$, then $F_{J}$ can be written as a convex combination of $F_{J_{1}}, \ldots, F_{J_{B}}$ multiplied by some phase factors $e^{i \Psi_{b}}$, see (3.12). We then employ an iterative procedure which estimates a carefully chosen norm of $F_{J}$ via the norms of $F_{J_{1}}, \ldots, F_{J_{B}}$. Each step in this procedure gives a gain $1-\varepsilon_{1}<1$ in the norm, and after $K$ steps we obtain a gain polynomial in $h$.

- To obtain a gain at each step, we consider two intervals $I \in V_{X}, J \in V_{Y}$ such that $|I| \cdot|J| \sim L h$, take their children $I_{1}, \ldots, I_{A}$ and $J_{1}, \ldots, J_{B}$, and argue similarly to Lemma 1.2 to show that the triangle inequality for $e^{i \Psi_{1}} J_{1}, \ldots, e^{i \Psi_{B}} J_{B}$ cannot be sharp on all the intervals $I_{1}, \ldots, I_{A}$.

- To do the latter, we take two pairs of children $I_{a}, I_{a}^{\prime}$ (with generic points in $I_{a}, I_{a}^{\prime}$ denoted $\left.x_{a}, x_{a^{\prime}}\right)$ and $J_{b}, J_{b}^{\prime}$. Due to the control on the derivatives of $F_{J_{b}}$, the differences $\left|F_{J_{b}}\left(x_{a}\right)-F_{J_{b}}\left(x_{a^{\prime}}\right)\right|$ and $\left|F_{J_{b^{\prime}}}\left(x_{a}\right)-F_{J_{b^{\prime}}}\left(x_{a^{\prime}}\right)\right|$ are bounded by $(L h)^{-1}|J| \cdot\left|x_{a}-x_{a^{\prime}}\right|$. On the other hand, the phase shift $\tau$ from (1.7) equals

$$
\tau=\Psi_{b}\left(x_{a}\right)+\Psi_{b^{\prime}}\left(x_{a^{\prime}}\right)-\Psi_{b^{\prime}}\left(x_{a}\right)-\Psi_{b}\left(x_{a^{\prime}}\right) \sim h^{-1}\left(x_{a}-x_{a^{\prime}}\right)\left(y_{b}-y_{b^{\prime}}\right) .
$$

Choosing $a, a^{\prime}, b, b^{\prime}$ such that $\left|x_{a}-x_{a^{\prime}}\right| \sim L^{-2 / 3}|I|,\left|y_{b}-y_{b^{\prime}}\right| \sim L^{-2 / 3}|J|$, and recalling that $|I| \cdot|J| \sim L h$, we see that $\tau \sim L^{-1 / 3}$ does not lie in $2 \pi \mathbb{Z}$ and it is larger than $(L h)^{-1}|J| \cdot\left|x_{a}-x_{a^{\prime}}\right| \sim L^{-2 / 3}$. This gives the necessary improvement on each step. Keeping track of the parameters in the argument, we obtain the bound (1.5) on $\varepsilon_{0}$.

This argument has many similarities with the method of Dolgopyat mentioned above. In particular, an inductive argument using $L^{2}$ norms appears for instance in $[\mathrm{Na} 05$, Lemma 5.4], which also features the spaces $\mathcal{C}_{\theta}$. The choice of children $I_{a}, I_{a^{\prime}}, J_{b}, J_{b^{\prime}}$ in the last step above is similar to the non local integrability condition (NLIC), see for instance $[\mathrm{Na0} 5, \S \S 2,5.3]$. However, our inductive Lemma 3.2 avoids the use of Dolgopyat operators and dense subsets (see for instance [Na05, p.138]), instead relying on strict convexity of balls in Hilbert spaces (see Lemma 2.7).

Moreover, the strategy of obtaining an essential spectral gap for hyperbolic surfaces in the present paper is significantly different from that of [Na05]. The latter uses zeta function techniques to reduce the spectral gap question to a spectral radius bound of a Ruelle transfer operator of the Bowen-Series map associated to the surface. The present paper instead relies on microlocal analysis of the scattering resolvent in [DyZa16] to reduce the gap problem to a fractal uncertainty principle, thus decoupling the dynamical aspects of the problem from the combinatorial ones. The role of the group invariance of the limit set, used in [Na05], is played here by its $\delta$-regularity (proved by Sullivan [Su79]), and words in the group are replaced by vertices in the discretizing tree. 


\subsection{Structure of the paper.}

- In $\S 2$, we establish basic properties of Ahlfors-David regular sets (§2.1), introduce the functional spaces used ( $\$ 2.2)$, and show several basic identities and inequalities ( $(2.3)$.

- In $\S 3$, we prove Theorem 1.

- In $\S 4$, we apply Theorem 1 and the results of [DyZa16] to establish an essential spectral gap for convex co-compact hyperbolic surfaces.

- In $\S 5$, we apply Theorem 1 and the results of [DyJi17, BoDy16] to establish an essential spectral gap for open quantum baker's maps.

\section{Preliminaries}

2.1. Regular sets and discretization. An interval in $\mathbb{R}$ is a subset of the form $I=[c, d]$ where $c<d$. Define the center of $I$ by $\frac{c+d}{2}$ and the size of $I$ by $|I|=d-c$.

Let $\mu$ be a finite measure on $\mathbb{R}$ with compact support. Fix an integer $L \geq 2$. Following [DyZa16, §6.4], we describe the discretization of $\mu$ with base L. For each $k \in \mathbb{Z}$, let $V_{k}$ be the set of all intervals $I=[c, d]$ which satisfy the following conditions:

- $c, d \in L^{-k} \mathbb{Z}$

- for each $q \in L^{-k} \mathbb{Z}$ with $c \leq q<d$, we have $\mu\left(\left[q, q+L^{-k}\right]\right)>0$;

- $\mu_{X}\left(\left[c-L^{-k}, c\right]\right)=\mu\left(\left[d, d+L^{-k}\right]\right)=0$.

In other words, $V_{k}$ is obtained by partitioning $\mathbb{R}$ into intervals of size $L^{-k}$, throwing out intervals of zero measure $\mu$, and merging consecutive intervals.

We define the set of vertices of the discretization as

$$
V:=\bigsqcup_{k \in \mathbb{Z}} V_{k},
$$

and define the height function by putting $H(I):=k$ if $I \in V_{k}$. (It is possible that $V_{k}$ intersect for different $k$, so formally speaking, a vertex is a pair $(k, I)$ where $I \in V_{k}$.) We say that $I \in V_{k}$ is a parent of $I^{\prime} \in V_{k+1}$, and $I^{\prime}$ is a child of $I$, if $I^{\prime} \subset I$. It is easy to check that the resulting structure has the following properties:

- any two distinct intervals $I, I^{\prime} \in V_{k}$ are at least $L^{-k}$ apart;

- $\mu\left(\mathbb{R} \backslash \bigsqcup_{I \in V_{k}} I\right)=0$ for all $k$;

- each $I \in V_{k}$ has exactly one parent;

- if $I \in V_{k}$ and $I_{1}, \ldots, I_{n} \in V_{k+1}$ are the children of $I$, then

$$
0<\mu(I)=\sum_{j=1}^{n} \mu\left(I_{j}\right) .
$$

For regular sets, the discretization has the following additional properties: 
Lemma 2.1. Let $L \geq 2, K>0$ be integers and assume that $\left(X, \mu_{X}\right)$ is $\delta$-regular up to scale $L^{-K}$ with regularity constant $C_{R}$, where $0<\delta<1$. Then the discretization of $\mu_{X}$ with base $L$ has the following properties:

1. Each $I \in V$ with $0 \leq H(I) \leq K$ satisfies for $C_{R}^{\prime}:=\left(3 C_{R}^{2}\right)^{\frac{1}{1-\delta}}$,

$$
\begin{gathered}
L^{-H(I)} \leq|I| \leq C_{R}^{\prime} L^{-H(I)} \\
C_{R}^{-1} L^{-\delta H(I)} \leq \mu_{X}(I) \leq C_{R}\left(C_{R}^{\prime}\right)^{\delta} L^{-\delta H(I)} .
\end{gathered}
$$

2. If $I^{\prime}$ is a child of $I \in V$ and $0 \leq H(I)<K$, then

$$
\frac{\mu_{X}\left(I^{\prime}\right)}{\mu_{X}(I)} \geq \frac{L^{-\delta}}{C_{R}^{\prime}}
$$

3. Assume that

$$
L \geq\left(4 C_{R}\right)^{\frac{6}{\delta(1-\delta)}} .
$$

Then for each $I \in V$ with $0 \leq H(I)<K$, there exist two children $I^{\prime}, I^{\prime \prime}$ of $I$ such that

$$
\frac{1}{2} C_{R}^{-2 / \delta} L^{-H(I)-2 / 3} \leq\left|x^{\prime}-x^{\prime \prime}\right| \leq 2 L^{-H(I)-2 / 3} \quad \text { for all } x^{\prime} \in I^{\prime}, x^{\prime \prime} \in I^{\prime \prime} .
$$

Remark. Parts 1 and 2 of the lemma state that the tree of intervals discretizing $\mu_{X}$ is approximately regular. Part 3, which is used at the end of $\S 3.2$, states that once the base of discretization $L$ is large enough, each interval $I$ in the tree has two children which are $\sim L^{-H(I)-2 / 3}$ apart from each other. A similar statement would hold if $2 / 3$ were replaced by any number in $(0,1)$.

Proof. 1. Put $k:=H(I)$. The lower bound on $|I|$ follows from the construction of the discretization. To show the upper bound, assume that $I=[c, d]$ and $d-c=M L^{-k}$. For each $q \in L^{-k} \mathbb{Z}$ with $c \leq q<d$, we have $\mu_{X}\left(\left[q, q+L^{-k}\right]\right)>0$, thus there exists $x_{q} \in\left[q, q+L^{-k}\right] \cap X$. Let $I_{q}$ be the interval of size $L^{-k}$ centered at $x_{q}$, see Figure 1 . Then

$$
\mu_{X}\left(\bigcup_{q} I_{q}\right) \leq \mu_{X}\left(\left[c-L^{-k}, d+L^{-k}\right]\right)=\mu_{X}(I) \leq C_{R}\left(M L^{-k}\right)^{\delta} .
$$

On the other hand, each point is covered by at most 3 intervals $I_{q}$, therefore

$$
M C_{R}^{-1} L^{-k \delta} \leq \sum_{q} \mu_{X}\left(I_{q}\right) \leq 3 \mu_{X}\left(\bigcup_{q} I_{q}\right) .
$$

Together these two inequalities imply that $M \leq C_{R}^{\prime}$, giving (2.2).

The upper bound on $\mu_{X}(I)$ follows from (2.2). To show the lower bound, take $x \in I \cap X$ and let $I^{\prime}$ be the interval of size $L^{-k}$ centered at $x$. Then $\mu_{X}\left(I^{\prime} \backslash I\right)=0$, therefore $\mu_{X}(I) \geq \mu_{X}\left(I^{\prime}\right) \geq C_{R}^{-1} L^{-\delta k}$.

2. This follows directly from $(2.3)$ and the fact that $C_{R}^{2}\left(C_{R}^{\prime}\right)^{\delta} \leq C_{R}^{\prime}$. 


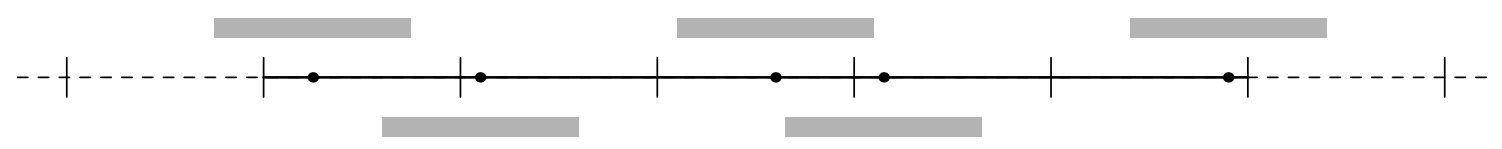

Figure 1. An illustration of the proof of the upper bound in (2.2). The ticks mark points in $L^{-k} \mathbb{Z}$, the solid interval is $I$, the dots mark the points $x_{q}$, and the shaded intervals are $I_{q}$. The intervals of length $L^{-k}$ adjacent to $I$ have zero measure $\mu_{X}$.

3. Put $k:=H(I)$. Take $x \in I \cap X$ and let $J$ be the interval of size $L^{-k-2 / 3}$ centered at $x$. Let $I_{1}, \ldots, I_{n}$ be all the intervals in $V_{k+1}$ which intersect $J$; they all have to be children of $I$. Let $x_{1}, \ldots, x_{n}$ be the centers of $I_{1}, \ldots, I_{n}$. Define

$$
T:=L^{k+2 / 3} \max _{j, \ell}\left|x_{j}-x_{\ell}\right| .
$$

By (2.2), we have $\left|I_{j}\right| \leq C_{R}^{\prime} L^{-k-1}$ and thus $T \leq 1+C_{R}^{\prime} L^{-1 / 3}$. On the other hand, the union of $I_{1}, \ldots, I_{n}$ is contained in an interval of size $T L^{-k-2 / 3}+C_{R}^{\prime} L^{-k-1}$. Therefore

$$
C_{R}^{-1} L^{-\delta(k+2 / 3)} \leq \mu_{X}(J) \leq \sum_{j=1}^{n} \mu_{X}\left(I_{j}\right) \leq C_{R}\left(T L^{-k-2 / 3}+C_{R}^{\prime} L^{-k-1}\right)^{\delta} .
$$

This implies that $T \geq C_{R}^{-2 / \delta}-C_{R}^{\prime} L^{-1 / 3}$.

Now, put $I^{\prime}:=I_{j}, I^{\prime \prime}=I_{\ell}$ where $j, \ell$ are chosen so that $T=L^{k+2 / 3}\left|x_{j}-x_{\ell}\right|$. Then for each $x^{\prime} \in I^{\prime}, x^{\prime \prime} \in I^{\prime \prime}$, we have by (2.5)

$$
\frac{1}{2} C_{R}^{-2 / \delta} \leq C_{R}^{-2 / \delta}-2 C_{R}^{\prime} L^{-1 / 3} \leq L^{k+2 / 3}\left|x^{\prime}-x^{\prime \prime}\right| \leq 1+2 C_{R}^{\prime} L^{-1 / 3} \leq 2
$$

which finishes the proof.

We finally the following estimates on Lebesgue measure of neighborhoods of $\delta$-regular set which are used in $\S \S 4,5$ :

Lemma 2.2. Assume that $\left(\Lambda, \mu_{\Lambda}\right)$ is $\delta$-regular up to scale $h \in(0,1)$ with constant $C_{R}$. Let $X:=\Lambda(h)=\Lambda+[-h, h]$ be the $h$-neighborhood of $\Lambda$ and define the measure $\mu_{X}$ by

$$
\mu_{X}(A):=h^{\delta-1} \mu_{L}(X \cap A), \quad A \subset \mathbb{R},
$$

where $\mu_{L}$ denotes the Lebesgue measure. Then $\left(X, \mu_{X}\right)$ is $\delta$-regular up to scale $h$ with constant $C_{R}^{\prime}:=30 C_{R}^{2}$.

Proof. We follow [DyZa16, Lemma 7.4]. Let $I \subset \mathbb{R}$ be an interval with $|I| \geq h$. Let $x_{1}, \ldots, x_{N} \in \Lambda \cap I(h)$ be a maximal set of $2 h$-separated points. Denote by $I_{n}^{\prime}$ the interval of size $h$ centered at $x_{n}$. Since $I_{n}^{\prime}$ are disjoint and their union is contained in 
$I(2 h)$, which is an interval of size $|I|+4 h \leq 5|I|$, we have

$$
N \cdot C_{R}^{-1} h^{\delta} \leq \sum_{n=1}^{N} \mu_{\Lambda}\left(I_{n}^{\prime}\right) \leq \mu_{\Lambda}(I(2 h)) \leq 5 C_{R}|I|^{\delta} .
$$

Next, let $I_{n}$ be the interval of size $6 h$ centered at $x_{n}$. Then $X \cap I$ is contained in the union of $I_{n}$ and thus

$$
\mu_{L}(X \cap I) \leq \sum_{n=1}^{N} \mu_{L}\left(I_{n}\right)=6 h N
$$

Together (2.7) and (2.8) give the required upper bound

$$
\mu_{X}(I)=h^{\delta-1} \mu_{L}(X \cap I) \leq 30 C_{R}^{2}|I|^{\delta} .
$$

Now, assume additionally that $|I| \leq 1$ and $I$ is centered at a point in $X$. Let $y_{1}, \ldots, y_{M} \in \Lambda \cap I$ be a maximal set of $h$-separated points. Denote by $I_{m}$ the interval of size $2 h$ centered at $y_{m}$. Then $\Lambda \cap I$ is contained in the union of $I_{m}$, therefore

$$
C_{R}^{-1}|I|^{\delta} \leq \mu_{\Lambda}(I)=\mu_{\Lambda}(\Lambda \cap I) \leq \sum_{m=1}^{M} \mu_{\Lambda}\left(I_{m}\right) \leq M \cdot 2 C_{R} h^{\delta} .
$$

Next, let $I_{m}^{\prime}$ be the interval of size $h$ centered at $y_{m}$. Then $I_{m}^{\prime} \subset X$ are nonoverlapping and each $I_{m}^{\prime} \cap I$ has size at least $h / 2$, therefore

$$
\mu_{L}(X \cap I) \geq \sum_{m=1}^{M} \mu_{L}\left(I_{m}^{\prime} \cap I\right) \geq M h / 2 .
$$

Combining (2.9) and (2.10) gives the required lower bound

$$
\mu_{X}(I)=h^{\delta-1} \mu_{L}(X \cap I) \geq \frac{1}{4 C_{R}^{2}}|I|^{\delta} .
$$

and finishes the proof.

2.2. Functional spaces. For a constant $\theta>0$ and an interval $I$, let $\mathcal{C}_{\theta}(I)$ be the space $C^{1}(I)$ with the norm

$$
\|f\|_{\mathcal{C}_{\theta}(I)}:=\max \left(\sup _{I}|f|, \theta|I| \cdot \sup _{I}\left|f^{\prime}\right|\right) .
$$

The following lemma shows that multiplications by functions of the form $\exp (i \psi)$ have norm 1 when mapping $\mathcal{C}_{\theta}(I)$ into the corresponding space for a sufficiently small subinterval of $I$ :

Lemma 2.3. Consider intervals

$$
I^{\prime} \subset I, \quad\left|I^{\prime}\right| \leq \frac{|I|}{4} .
$$


Assume that $\psi \in C^{\infty}(I ; \mathbb{R})$ and $\theta>0$ are such that

$$
4 \theta\left|I^{\prime}\right| \cdot \sup _{I^{\prime}}\left|\psi^{\prime}\right| \leq 1
$$

Then for each $f \in \mathcal{C}_{\theta}(I)$, we have $\|\exp (i \psi) f\|_{\mathcal{C}_{\theta}\left(I^{\prime}\right)} \leq\|f\|_{\mathcal{C}_{\theta}(I)}$ and

$$
\theta\left|I^{\prime}\right| \cdot \sup _{I^{\prime}}\left|(\exp (i \psi) f)^{\prime}\right| \leq \frac{\|f\|_{\mathcal{C}_{\theta}(I)}}{2}
$$

Proof. The left-hand side of (2.13) is bounded from above by

$$
\theta\left|I^{\prime}\right| \cdot\left(\sup _{I^{\prime}}\left|\psi^{\prime} f\right|+\sup _{I^{\prime}}\left|f^{\prime}\right|\right) .
$$

From (2.12), (2.11) we get

$$
\theta\left|I^{\prime}\right| \cdot \sup _{I^{\prime}}\left|\psi^{\prime} f\right| \leq \frac{\|f\|_{\mathcal{C}_{\theta}(I)}}{4}, \quad \theta\left|I^{\prime}\right| \cdot \sup _{I^{\prime}}\left|f^{\prime}\right| \leq \frac{\|f\|_{\mathcal{C}_{\theta}(I)}}{4}
$$

which finishes the proof of (2.13). The bound (2.13) implies that $\|\exp (i \psi) f\|_{\mathcal{C}_{\theta}\left(I^{\prime}\right)} \leq$ $\|f\|_{\mathcal{C}_{\theta}(I)}$.

The following is a direct consequence of the mean value theorem:

Lemma 2.4. Let $f \in \mathcal{C}_{\theta}(I)$. Then for all $x, x^{\prime} \in I$, we have

$$
\left|f(x)-f\left(x^{\prime}\right)\right| \leq \frac{\left|x-x^{\prime}\right|}{\theta|I|} \cdot\|f\|_{\mathcal{C}_{\theta}(I)} .
$$

2.3. A few technical lemmas. The following is a two-dimensional analogue of the mean value theorem:

Lemma 2.5. Let $I=\left[c_{1}, d_{1}\right]$ and $J=\left[c_{2}, d_{2}\right]$ be two intervals and $\Phi \in C^{2}(I \times J ; \mathbb{R})$. Then there exists $\left(x_{0}, y_{0}\right) \in I \times J$ such that

$$
\Phi\left(c_{1}, c_{2}\right)+\Phi\left(d_{1}, d_{2}\right)-\Phi\left(c_{1}, d_{2}\right)-\Phi\left(d_{1}, c_{2}\right)=|I| \cdot|J| \cdot \partial_{x y}^{2} \Phi\left(x_{0}, y_{0}\right) .
$$

Proof. Replacing $\Phi(x, y)$ by $\Phi(x, y)-\Phi\left(c_{1}, y\right)-\Phi\left(x, c_{2}\right)+\Phi\left(c_{1}, c_{2}\right)$, we may assume that $\Phi\left(c_{1}, y\right)=0$ and $\Phi\left(x, c_{2}\right)=0$ for all $x \in I, y \in J$. By the mean value theorem, we have $\Phi\left(d_{1}, d_{2}\right)=|I| \cdot \partial_{x} \Phi\left(x_{0}, d_{2}\right)$ for some $x_{0} \in I$. Applying the mean value theorem again, we have $\partial_{x} \Phi\left(x_{0}, d_{2}\right)=|J| \cdot \partial_{x y}^{2} \Phi\left(x_{0}, y_{0}\right)$ for some $y_{0} \in J$, finishing the proof.

Lemma 2.6. Assume that $\tau \in \mathbb{R}$ and $|\tau| \leq \pi$. Then $\left|e^{i \tau}-1\right| \geq \frac{2}{\pi}|\tau|$.

Proof. We have $\left|e^{i \tau}-1\right|=2 \sin (|\tau| / 2)$. It remains to use that $\sin x \geq \frac{2}{\pi} x$ when $0 \leq x \leq \frac{\pi}{2}$, which follows from the concavity of $\sin x$ on that interval.

The next lemma, used several times in $\S 3.2$, is a quantitative version of the fact that balls in Hilbert spaces are strictly convex: 
Lemma 2.7. Assume that $\mathcal{H}$ is a Hilbert space, $f_{1}, \ldots, f_{n} \in \mathcal{H}, p_{1}, \ldots, p_{n} \geq 0$, and $p_{1}+\cdots+p_{n}=1$. Then

$$
\left\|\sum_{j=1}^{n} p_{j} f_{j}\right\|_{\mathcal{H}}^{2}=\sum_{j=1}^{n} p_{j}\left\|f_{j}\right\|_{\mathcal{H}}^{2}-\sum_{1 \leq j<\ell \leq n} p_{j} p_{\ell}\left\|f_{j}-f_{\ell}\right\|_{\mathcal{H}}^{2} \cdot
$$

If moreover for some $\varepsilon, R \geq 0$

$$
\sum_{j=1}^{n} p_{j}\left\|f_{j}\right\|_{\mathcal{H}}^{2}=R, \quad\left\|\sum_{j=1}^{n} p_{j} f_{j}\right\|_{\mathcal{H}}^{2} \geq(1-\varepsilon) R, \quad p_{\min }:=\min _{j} p_{j} \geq 2 \sqrt{\varepsilon}
$$

then for all $j$

$$
\frac{\sqrt{R}}{2} \leq\left\|f_{j}\right\|_{\mathcal{H}} \leq 2 \sqrt{R}
$$

Proof. The identity (2.15) follows by a direct computation. To show (2.17), note that by (2.15) and (2.16) for each $j, \ell$

$$
\left\|f_{j}-f_{\ell}\right\|_{\mathcal{H}}^{2} \leq \frac{\varepsilon R}{p_{\min }^{2}} \leq \frac{R}{4}
$$

Put $f_{\max }:=\max _{j}\left\|f_{j}\right\|_{\mathcal{H}}$ and $f_{\min }:=\min _{j}\left\|f_{j}\right\|_{\mathcal{H}}$, then

$$
f_{\max }-f_{\min } \leq \frac{\sqrt{R}}{2}, \quad f_{\min } \leq \sqrt{R} \leq f_{\max }
$$

which implies (2.17).

Lemma 2.8. Assume that $\alpha_{j}, p_{j} \geq 0, j=1, \ldots, n, p_{1}+\cdots+p_{n}=1$, and for some $\varepsilon, R \geq 0$

$$
\sum_{j=1}^{n} p_{j} \alpha_{j} \geq(1-\varepsilon) R, \quad \max _{j} \alpha_{j} \leq R, \quad p_{\min }:=\min _{j} p_{j} \geq 2 \varepsilon .
$$

Then for all $j$,

$$
\alpha_{j} \geq \frac{R}{2}
$$

Proof. We have

$$
\sum_{j=1}^{n} p_{j}\left(R-\alpha_{j}\right) \leq \varepsilon R .
$$

All the terms in the sum are nonnegative, therefore for all $j$

$$
R-\alpha_{j} \leq \frac{\varepsilon R}{p_{\min }} \leq \frac{R}{2}
$$

finishing the proof. 


\section{Proof of Theorem 1}

3.1. The iterative argument. In this section, we prove the following statement which can be viewed as a special case of Theorem 1. Its proof relies on an inductive bound, Lemma 3.2, which is proved in §3.2. In §3.3, we deduce Theorem 1 from Proposition 3.1, in particular removing the condition (3.1).

Proposition 3.1. Let $\delta, \delta^{\prime} \in(0,1), C_{R}>1, I_{0}, J_{0} \subset \mathbb{R}$ be some intervals, $G \in$ $C^{1}\left(I_{0} \times J_{0} ; \mathbb{C}\right)$, and the phase function $\Phi \in C^{2}\left(I_{0} \times J_{0} ; \mathbb{R}\right)$ satisfy

$$
\frac{1}{2}<\left|\partial_{x y}^{2} \Phi(x, y)\right|<2 \quad \text { for all }(x, y) \in I_{0} \times J_{0} .
$$

Choose constants $C_{R}^{\prime}>0$ and $L \in \mathbb{N}$ such that

$$
C_{R}^{\prime}=\left(2 C_{R}\right)^{\frac{2}{1-\max \left(\delta, \delta^{\prime}\right)}}, \quad L \geq\left(2 C_{R}^{\prime}\left(6 C_{R}\right)^{\frac{1}{\delta}+\frac{1}{\delta^{\prime}}}\right)^{6} .
$$

Fix $K_{0} \in \mathbb{N}_{0}$ and put $h:=L^{-K}$ for some $K \in \mathbb{N}_{0}, K \geq 2 K_{0}$. Assume that $\left(X, \mu_{X}\right)$ is $\delta$-regular, and $\left(Y, \mu_{Y}\right)$ is $\delta^{\prime}$-regular, up to scale $L^{K_{0}-K}$ with regularity constant $C_{R}$, and $X \subset I_{0}, Y \subset J_{0}$. Put

$$
\varepsilon_{1}:=10^{-5}\left(C_{R}^{\frac{1}{\delta}+\frac{1}{\delta^{\prime}}} C_{R}^{\prime}\right)^{-4} L^{-5}, \quad \varepsilon_{0}:=-\frac{\log \left(1-\varepsilon_{1}\right)}{2 \log L} .
$$

Then for some $C$ depending only on $K_{0}, G, \mu_{X}(X), \mu_{Y}(Y)$, and $\mathcal{B}_{h}$ defined in (1.3),

$$
\left\|\mathcal{B}_{h} f\right\|_{L^{2}\left(X, \mu_{X}\right)} \leq C h^{\varepsilon_{0}}\|f\|_{L^{2}\left(Y, \mu_{Y}\right)} \quad \text { for all } f \in L^{2}\left(Y, \mu_{Y}\right)
$$

Remark. Proposition 3.1 has complicated hypotheses in order to make it useful for the proof of Theorem 1. However, the argument is essentially the same in the following special case which could simplify the reading of the proof below: $\delta=\delta^{\prime}, G \equiv 1$, $\Phi(x, y)=x y, K_{0}=0$. Note that in this case $\mathcal{B}_{h}$ is related to the semiclassical Fourier transform (1.2).

To start the proof of Proposition 3.1, we extend $\Phi$ to a function in $C^{2}\left(\mathbb{R}^{2} ; \mathbb{R}\right)$ such that (3.1) still holds, and extend $G$ to a function in $C^{1}\left(\mathbb{R}^{2} ; \mathbb{C}\right)$ such that $G, \partial_{x} G$ are uniformly bounded. Following $§ 2.1$ consider the discretizations of $\mu_{X}, \mu_{Y}$ with base $L$, denoting by $V_{X}, V_{Y}$ the sets of vertices and by $H$ the height functions.

Fix $f \in L^{2}\left(Y, \mu_{Y}\right)$. For each $J \in V_{Y}$, let $y_{J}$ denote the center of $J$ and define the function of $x \in \mathbb{R}$,

$$
F_{J}(x)=\frac{1}{\mu_{Y}(J)} \int_{J} \exp \left(\frac{i\left(\Phi(x, y)-\Phi\left(x, y_{J}\right)\right)}{h}\right) G(x, y) f(y) d \mu_{Y}(y) .
$$

In terms of the operator $\mathcal{B}_{h}$ from (1.3), we may write

$$
F_{J}(x)=\frac{1}{\mu_{Y}(J)} \exp \left(-\frac{i \Phi\left(x, y_{J}\right)}{h}\right) \mathcal{B}_{h}\left(\mathbb{1}_{J} f\right)(x) .
$$



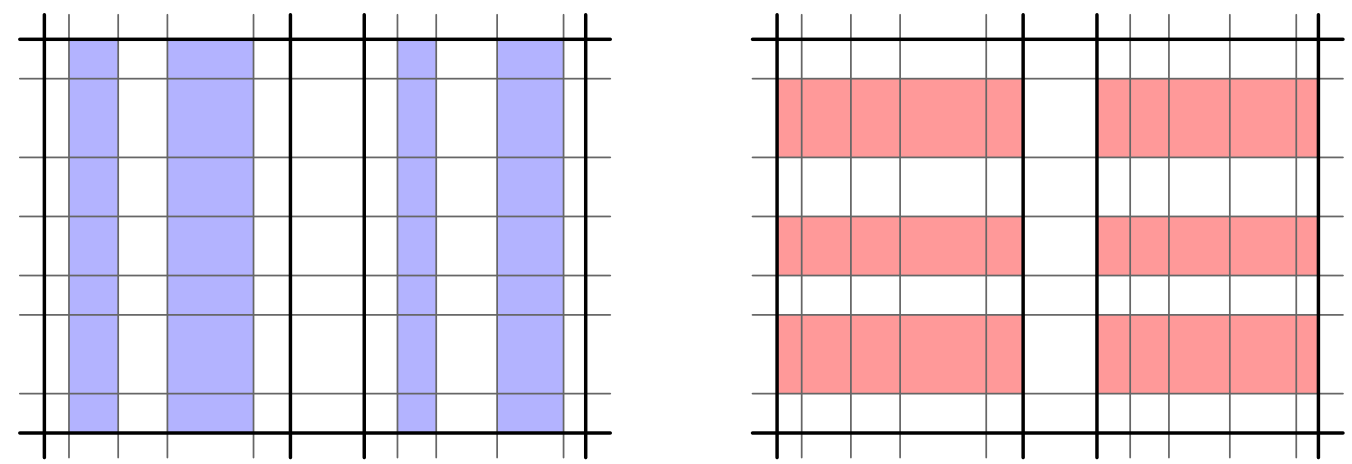

Figure 2. An illustration of (3.8) in the case $K=1$. The vertical lines mark the endpoints of intervals in $V_{X}$ and the horizontal lines, the endpoints of intervals in $V_{Y}$. The thick lines correspond to intervals of height 0 and the thin lines, to intervals of height 1 . The shaded rectangles have the form $I \times J, I \in V_{X}, J \in V_{Y}$, where $E_{J}$ is constant on $I$, and the shaded rectangles on the left/on the right correspond to the left/right hand sides of (3.9) for $H(J)=0$.

Put

$$
\theta:=\frac{1}{8\left(C_{R}^{\prime}\right)^{2}}
$$

and for $J \in V_{Y}$ define the piecewise constant function $E_{J} \in L^{\infty}\left(X, \mu_{X}\right)$ using the space $\mathcal{C}_{\theta}(I)$ defined in $\S 2.2$ :

$$
E_{J}(x)=\left\|F_{J}\right\|_{\mathcal{C}_{\theta}(I)} \quad \text { where } \quad x \in I \in V_{X}, H(I)+H(J)=K .
$$

See Figure 2. Note that $\left|F_{J}(x)\right| \leq E_{J}(x)$ for $\mu_{X}$-almost every $x$.

The $L^{2}$ norms of the functions $E_{J}$ satisfy the following key bound, proved in $§ 3.2$, which gives an improvement from one scale to the next. The use of the $L^{2}$ norm of $E_{J}$ as the monotone quantity is convenient for several reasons. On one hand, the averaging provided by the $L^{2}$ norm means it is only necessary to show an improvement on $F_{J}$ in sufficiently many places; more precisely we will show in (3.11) that such improvement happens on at least one child of each interval $I \in V(X)$ with $H(I)+H(J)=K-1$. On the other hand, such improvement is obtained by a pointwise argument which also uses that $F_{J_{b}}$ are slowly varying on each interval $I$ with $H(I)+H(J)=K-1$ (see Lemma 3.7); this motivates the use of $\mathcal{C}_{\theta}(I)$ norms in the definition of $E_{J_{b}}$.

Lemma 3.2. Let $J \in V_{Y}$ with $K_{0} \leq H(J)<K-K_{0}$ and $J_{1}, \ldots, J_{B} \in V_{Y}$ be the children of $J$. Then, with $\varepsilon_{1}$ defined in (3.3),

$$
\left\|E_{J}\right\|_{L^{2}\left(X, \mu_{X}\right)}^{2} \leq\left(1-\varepsilon_{1}\right) \sum_{b=1}^{B} \frac{\mu_{Y}\left(J_{b}\right)}{\mu_{Y}(J)}\left\|E_{J_{b}}\right\|_{L^{2}\left(X, \mu_{X}\right)}^{2} .
$$

Iterating Lemma 3.2, we obtain 
Proof of Proposition 3.1. First of all, we show that for all $J \in V_{Y}$ with $H(J)=K-K_{0}$, and some constant $C_{0}$ depending on $G, \mu_{X}(X)$ and defined below

$$
\left\|E_{J}\right\|_{L^{2}\left(X, \mu_{X}\right)}^{2} \leq C_{0} \frac{\|f\|_{L^{2}\left(J, \mu_{Y}\right)}^{2}}{\mu_{Y}(J)}
$$

Indeed, take $I \in V_{X}$ such that $H(I)=K_{0}$. By (2.2) and (3.1), for all $y \in J$

$$
\frac{1}{h} \sup _{x \in I}\left|\partial_{x} \Phi(x, y)-\partial_{x} \Phi\left(x, y_{J}\right)\right| \leq \frac{2}{h}|J| \leq 2 C_{R}^{\prime} L^{K_{0}}
$$

and thus by (2.2) and (3.7)

$$
\frac{4 \theta|I|}{h} \sup _{x \in I}\left|\partial_{x} \Phi(x, y)-\partial_{x} \Phi\left(x, y_{J}\right)\right| \leq 1
$$

Arguing similarly to Lemma 2.3, we obtain for all $y \in J$

$$
\left\|\exp \left(\frac{i\left(\Phi(x, y)-\Phi\left(x, y_{J}\right)\right)}{h}\right) G(x, y)\right\|_{\mathcal{C}_{\theta}(I)} \leq C_{G}:=\max \left(\sup |G|, \sup \left|\partial_{x} G\right|\right) \text {. }
$$

Using Hölder's inequality in (3.5), we obtain

$$
\left.E_{J}\right|_{I}=\left\|F_{J}\right\|_{\mathcal{C}_{\theta}(I)} \leq \frac{C_{G}}{\mu_{Y}(J)} \int_{J}|f(y)| d \mu_{Y}(y) \leq \frac{C_{G}\|f\|_{L^{2}\left(J, \mu_{Y}\right)}}{\sqrt{\mu_{Y}(J)}}
$$

and (3.10) follows by integration in $x$, where we put $C_{0}:=C_{G}^{2} \mu_{X}(X)$.

Now, arguing by induction on $H(J)$ with (3.10) as base and (3.9) as inductive step, we obtain for all $J \in V_{Y}$ with $K_{0} \leq H(J) \leq K-K_{0}$,

$$
\left\|E_{J}\right\|_{L^{2}\left(X, \mu_{X}\right)}^{2} \leq C_{0}\left(1-\varepsilon_{1}\right)^{K-K_{0}-H(J)} \frac{\|f\|_{L^{2}\left(J, \mu_{Y}\right)}^{2}}{\mu_{Y}(J)}
$$

In particular, for all $J \in V_{Y}$ with $H(J)=K_{0}$, we have by (3.6)

$$
\left\|\frac{\mathcal{B}_{h}\left(\mathbb{1}_{J} f\right)}{\mu_{Y}(J)}\right\|_{L^{2}\left(X, \mu_{X}\right)}^{2}=\left\|F_{J}\right\|_{L^{2}\left(X, \mu_{X}\right)}^{2} \leq\left\|E_{J}\right\|_{L^{2}\left(X, \mu_{X}\right)}^{2} \leq C_{1} h^{2 \varepsilon_{0}} \frac{\|f\|_{L^{2}\left(J, \mu_{Y}\right)}^{2}}{\mu_{Y}(J)} .
$$

where $C_{1}:=C_{0}\left(1-\varepsilon_{1}\right)^{-2 K_{0}}$. Using the identity

$$
\mathcal{B}_{h} f=\mu_{Y}(Y) \sum_{J \in V_{Y}, H(J)=K_{0}} \frac{\mu_{Y}(J)}{\mu_{Y}(Y)} \cdot \frac{\mathcal{B}_{h}\left(\mathbb{1}_{J} f\right)}{\mu_{Y}(J)}
$$

and (2.15), we estimate

$$
\left\|\mathcal{B}_{h} f\right\|_{L^{2}\left(X, \mu_{X}\right)}^{2} \leq C_{1} \mu_{Y}(Y) h^{2 \varepsilon_{0}}\|f\|_{L^{2}\left(Y, \mu_{Y}\right)}^{2}
$$

and (3.4) follows with $C:=C_{G}\left(1-\varepsilon_{1}\right)^{-K_{0}} \sqrt{\mu_{X}(X) \mu_{Y}(Y)}$. 
3.2. The inductive step. In this section we prove Lemma 3.2. Let $J \in V_{Y}$ satisfy $K_{0} \leq H(J)<K-K_{0}$ and $J_{1}, \ldots, J_{B}$ be the children of $J$. It suffices to show that for all $I \in V_{X}$ with $H(I)+H(J)=K-1$ we have

$$
\left\|E_{J}\right\|_{L^{2}\left(I, \mu_{X}\right)}^{2} \leq\left(1-\varepsilon_{1}\right) \sum_{b=1}^{B} \frac{\mu_{Y}\left(J_{b}\right)}{\mu_{Y}(J)}\left\|E_{J_{b}}\right\|_{L^{2}\left(I, \mu_{X}\right)}^{2} .
$$

Indeed, summing (3.11) over $I$, we obtain (3.9).

Fix $I \in V_{X}$ with $H(I)+H(J)=K-1$ and let $I_{1}, \ldots, I_{A}$ be the children of $I$. Define

$$
p_{a}:=\frac{\mu_{X}\left(I_{a}\right)}{\mu_{X}(I)}, \quad q_{b}:=\frac{\mu_{Y}\left(J_{b}\right)}{\mu_{Y}(J)} .
$$

Note that $p_{a}, q_{b} \geq 0$ and $p_{1}+\cdots+p_{A}=q_{1}+\cdots+q_{B}=1$.

The functions $F_{J}$ and $F_{J_{b}}$ are related by the following formula:

$$
F_{J}=\sum_{b=1}^{B} q_{b} \exp \left(i \Psi_{b}\right) F_{J_{b}}, \quad \Psi_{b}(x):=\frac{\Phi\left(x, y_{J_{b}}\right)-\Phi\left(x, y_{J}\right)}{h} .
$$

That is, $F_{J}$ is a convex combination of $F_{J_{1}}, \ldots, F_{J_{B}}$ multiplied by the phase factors $\exp \left(i \Psi_{b}\right)$. At the end of this subsection we exploit cancellation between these phase factors to show (3.11). However there are several preparatory steps necessary. Before we proceed with the proof, we show the version of (3.11) with no improvement:

Lemma 3.3. We have

$$
\left\|E_{J}\right\|_{L^{2}\left(I, \mu_{X}\right)}^{2} \leq \sum_{b=1}^{B} q_{b}\left\|E_{J_{b}}\right\|_{L^{2}\left(I, \mu_{X}\right)}^{2}
$$

Proof. By (2.2), (3.1), and (3.7), we have for all $a, b$

$$
4 \theta\left|I_{a}\right| \cdot \sup _{I}\left|\Psi_{b}^{\prime}\right| \leq \frac{8 \theta\left|I_{a}\right| \cdot|J|}{h} \leq 1
$$

Moreover, by (2.2) and (3.2) we have $\left|I_{a}\right| \leq \frac{1}{4}|I|$. Applying Lemma 2.3, we obtain

$$
\left\|\exp \left(i \Psi_{b}\right) F_{J_{b}}\right\|_{C_{\theta}\left(I_{a}\right)} \leq\left\|F_{J_{b}}\right\|_{C_{\theta}(I)} .
$$

By (3.12) and (2.15) we then have

$$
\left\|F_{J}\right\|_{C_{\theta}\left(I_{a}\right)}^{2} \leq\left(\sum_{b=1}^{B} q_{b}\left\|F_{J_{b}}\right\|_{C_{\theta}(I)}\right)^{2} \leq \sum_{b=1}^{B} q_{b}\left\|F_{J_{b}}\right\|_{C_{\theta}(I)}^{2} .
$$

By (3.8), we have for all $a, b$

$$
\left.E_{J}\right|_{I_{a}}=\left\|F_{J}\right\|_{\mathcal{C}_{\theta}\left(I_{a}\right)},\left.\quad E_{J_{b}}\right|_{I}=\left\|F_{J_{b}}\right\|_{\mathcal{C}_{\theta}(I)} .
$$

Now, summing both sides of (3.15) over $a$ with weights $\mu_{X}\left(I_{a}\right)$, we obtain (3.13). 
The rest of this section is dedicated to the proof of (3.11), studying the situations in which the bound (3.13) is almost sharp and ultimately reaching a contradiction. The argument is similar in spirit to Lemma 1.2. In fact we can view Lemma 1.2 as the special degenerate case when $A=B=2, p_{a}=q_{b}=\frac{1}{2}$, the intervals $I_{a}$ are replaced by points $x_{a}, F_{J_{b}} \equiv f_{b}$ are constants, $u_{a}=F_{J}\left(x_{a}\right)$, and $\omega_{a b}=\Psi_{b}\left(x_{a}\right)$. The general case is more technically complicated. In particular we use Lemma 2.7 to deal with general convex combinations. We also use $\delta$-regularity in many places, for instance to show that the coefficients $p_{a}, q_{b}$ are bounded away from zero and to get the phase factor cancellations in (3.30) at the end of the proof. The reading of the argument below may be simplified by making the illegal choice $\varepsilon_{1}:=0$.

We henceforth assume that (3.11) does not hold. Put

$$
R:=\sum_{b=1}^{B} q_{b}\left\|F_{J_{b}}\right\|_{\mathcal{C}_{\theta}(I)}^{2}
$$

By (3.16), the failure of (3.11) can be rewritten as

$$
\sum_{a=1}^{A} p_{a}\left\|F_{J}\right\|_{\mathcal{C}_{\theta}\left(I_{a}\right)}^{2}>\left(1-\varepsilon_{1}\right) R
$$

We note for future use that $p_{a}, q_{b}$ are bounded below by (2.4):

$$
p_{\min }:=\min _{a} p_{a} \geq \frac{L^{-\delta}}{C_{R}^{\prime}}, \quad q_{\min }:=\min _{b} q_{b} \geq \frac{L^{-\delta^{\prime}}}{C_{R}^{\prime}} .
$$

We first deduce from (3.17) and the smallness of $\varepsilon_{1}$ an upper bound on each $\left\|F_{J_{b}}\right\|_{\mathcal{C}_{\theta}(I)}$ in terms of the averaged quantity $R$ :

Lemma 3.4. We have for all $b$,

$$
\left\|F_{J_{b}}\right\|_{\mathcal{C}_{\theta}(I)} \leq 2 \sqrt{R}
$$

Proof. The first inequality in (3.15) together with (3.18) implies

$$
\left(\sum_{b=1}^{B} q_{b}\left\|F_{J_{b}}\right\|_{\mathcal{C}_{\theta}(I)}\right)^{2} \geq \sum_{a=1}^{A} p_{a}\left\|F_{J}\right\|_{\mathcal{C}_{\theta}\left(I_{a}\right)}^{2} \geq\left(1-\varepsilon_{1}\right) R .
$$

By (3.3) and (3.19) we have $q_{\min } \geq 2 \sqrt{\varepsilon_{1}}$. Applying (2.17) to $f_{b}:=\left\|F_{J_{b}}\right\|_{\mathcal{C}_{\theta(I)}}$ with (3.17) and (3.21), we obtain (3.20).

We next obtain a version of (3.18) which gives a lower bound on the size of $F_{J}$, rather than on the norm $\left\|F_{J}\right\|_{\mathcal{C}_{\theta}\left(I_{a}\right)}$ :

Lemma 3.5. There exist $x_{a} \in I_{a}, a=1, \ldots, A$, such that

$$
\sum_{a=1}^{A} p_{a}\left|F_{J}\left(x_{a}\right)\right|^{2}>\left(1-2 \varepsilon_{1}\right) R
$$


Proof. By Lemma 2.3 and (3.14), we have

$$
\theta\left|I_{a}\right| \cdot \sup _{I_{a}}\left|\left(\exp \left(i \Psi_{b}\right) F_{J_{b}}\right)^{\prime}\right| \leq \frac{\left\|F_{J_{b}}\right\|_{\mathcal{C}_{\theta}(I)}}{2}
$$

It follows by (3.12) and the triangle inequality that for all $a$,

$$
\left\|F_{J}\right\|_{\mathcal{C}_{\theta}\left(I_{a}\right)} \leq \max \left(\sup _{I_{a}}\left|F_{J}\right|, \frac{1}{2} \sum_{b=1}^{B} q_{b}\left\|F_{J_{b}}\right\|_{\mathcal{C}_{\theta}(I)}\right) .
$$

By (3.15) we have

$$
\sup _{I_{a}}\left|F_{J}\right|^{2} \leq\left\|F_{J}\right\|_{\mathcal{C}_{\theta}\left(I_{a}\right)}^{2} \leq R
$$

Therefore by (3.23) and the second inequality in (3.15)

$$
\left\|F_{J}\right\|_{\mathcal{C}_{\theta}\left(I_{a}\right)}^{2} \leq \frac{1}{2}\left(R+\sup _{I_{a}}\left|F_{J}\right|^{2}\right)
$$

Summing this inequality over $a$ with weights $p_{a}$, we see that (3.18) implies

$$
\sum_{a=1}^{A} p_{a} \sup _{I_{a}}\left|F_{J}\right|^{2}>\left(1-2 \varepsilon_{1}\right) R
$$

which gives (3.22).

Now, choose $x_{a}$ as in Lemma 3.5 and put

$$
F_{a b}:=F_{J_{b}}\left(x_{a}\right) \in \mathbb{C}, \quad \omega_{a b}:=\Psi_{b}\left(x_{a}\right) \in \mathbb{R} .
$$

Note that by (3.12)

$$
F_{J}\left(x_{a}\right)=\sum_{b=1}^{B} q_{b} \exp \left(i \omega_{a b}\right) F_{a b}
$$

Using (2.15) for $f_{b}=\exp \left(i \omega_{a b}\right) F_{a b}$ and (3.22), we obtain

$$
\sum_{a, b} p_{a} q_{b}\left|F_{a b}\right|^{2}>\left(1-2 \varepsilon_{1}\right) R+\sum_{\substack{a, b, b^{\prime} \\ b<b^{\prime}}} p_{a} q_{b} q_{b^{\prime}}\left|\exp \left(i\left(\omega_{a b}-\omega_{a b^{\prime}}\right)\right) F_{a b}-F_{a b^{\prime}}\right|^{2} .
$$

From the definition (3.17) of $R$, we have for all $a$

$$
\sum_{b=1}^{B} q_{b}\left|F_{a b}\right|^{2} \leq R
$$

Therefore, the left-hand side of (3.25) is bounded above by $R$. Using (3.19), we then get for all $a, b, b^{\prime}$ the following approximate equality featuring the phase terms $\omega_{a b}$ :

$$
\left|\exp \left(i\left(\omega_{a b}-\omega_{a b^{\prime}}\right)\right) F_{a b}-F_{a b^{\prime}}\right|<\sqrt{\frac{2 \varepsilon_{1} R}{p_{\min } q_{\min }^{2}}} \leq 2\left(C_{R}^{\prime}\right)^{2} L^{\delta+\delta^{\prime}} \sqrt{\varepsilon_{1} R} .
$$

Using the smallness of $\varepsilon_{1}$, we obtain from here a lower bound on $\left|F_{a b}\right|$ : 
Lemma 3.6. For all $a, b$ we have

$$
\left|F_{a b}\right| \geq \frac{\sqrt{R}}{2}
$$

Proof. By (3.3) and (3.19), we have $p_{\min } \geq 4 \varepsilon_{1}$. Applying Lemma 2.8 to $\alpha_{a}=$ $\sum_{b} q_{b}\left|F_{a b}\right|^{2}$ and using (3.25) and (3.26), we obtain for all $a$

$$
\sum_{b=1}^{B} q_{b}\left|F_{a b}\right|^{2} \geq \frac{R}{2}
$$

We now argue similarly to the proof of (2.17). Fix $a$ and let $F_{a \text {,min }}=\min _{b}\left|F_{a b}\right|$, $F_{a, \max }=\max _{b}\left|F_{a b}\right|$. By (3.29) we have $F_{a, \max } \geq \sqrt{R / 2}$. On the other hand the difference $F_{a, \max }-F_{a \text {,min }}$ is bounded above by (3.27). By (3.3) we then have

$$
F_{a, \min } \geq \sqrt{\frac{R}{2}}-2\left(C_{R}^{\prime}\right)^{2} L^{\delta+\delta^{\prime}} \sqrt{\varepsilon_{1} R} \geq \frac{\sqrt{R}}{2},
$$

finishing the proof.

We next estimate the discrepancy between the values $F_{a b}$ for fixed $b$ and different $a$, using the fact that we control the norm $\left\|F_{J_{b}}\right\|_{\mathcal{C}_{\theta}(I)}$ and thus the derivative of $F_{J_{b}}$ :

Lemma 3.7. For all $a, a^{\prime}, b$ we have

$$
\left|F_{a b}-F_{a^{\prime} b}\right| \leq \frac{2 \sqrt{R}\left|x_{a}-x_{a^{\prime}}\right|}{\theta|I|} \leq \frac{2 \sqrt{R}}{\theta} L^{H(I)} \cdot\left|x_{a}-x_{a^{\prime}}\right| .
$$

Proof. This follows immediately by combining Lemma 2.4, Lemma 3.4, and (2.2).

Armed with the bounds obtained above, we are now ready to reach a contradiction and finish the proof of Lemma 3.2, using the discrepancy of the phase shifts $\omega_{a b}$ and the lower bound on $\left|\partial_{x y}^{2} \Phi\right|$ from (3.1).

Using part 3 of Lemma 2.1 and (3.2), choose $a, a^{\prime}, b, b^{\prime}$ such that

$$
\begin{aligned}
& \frac{1}{2} C_{R}^{-2 / \delta} L^{-2 / 3} \leq L^{H(I)} \cdot\left|x_{a}-x_{a^{\prime}}\right| \leq 2 L^{-2 / 3}, \\
& \frac{1}{2} C_{R}^{-2 / \delta^{\prime}} L^{-2 / 3} \leq L^{H(J)} \cdot\left|y_{b}-y_{b^{\prime}}\right| \leq 2 L^{-2 / 3} .
\end{aligned}
$$

Recall that $x_{a} \in I_{a}$ is chosen in Lemma 3.5 and $y_{b}:=y_{J_{b}}$ is the center of $J_{b}$. By Lemma 2.5, we have for some $(\tilde{x}, \tilde{y}) \in I \times J$,

$$
\tau:=\omega_{a b}+\omega_{a^{\prime} b^{\prime}}-\omega_{a^{\prime} b}-\omega_{a b^{\prime}}=\frac{\left(x_{a}-x_{a^{\prime}}\right)\left(y_{b}-y_{b^{\prime}}\right)}{h} \partial_{x y}^{2} \Phi(\tilde{x}, \tilde{y}) .
$$

By (3.1) and (3.2) and since $h=L^{-K}, H(I)+H(J)=K-1$, we have

$$
\frac{C_{R}^{-\frac{2}{\delta}-\frac{2}{\delta^{\prime}}}}{8} L^{-1 / 3} \leq|\tau| \leq 8 L^{-1 / 3} \leq \pi \text {. }
$$


Therefore, by Lemma 2.6 the phase factor $e^{i \tau}$ is bounded away from 1, which combined with (3.28) gives a lower bound on the discrepancy:

$$
\left|F_{a b}\right| \cdot\left|e^{i \tau}-1\right| \geq \frac{|\tau| \sqrt{R}}{\pi} \geq \frac{C_{R}^{-\frac{2}{\delta}-\frac{2}{\delta^{\prime}}}}{8 \pi} L^{-1 / 3} \sqrt{R} .
$$

On the other hand we can estimate the same discrepancy from above by (3.27), Lemma 3.7, and the triangle inequality:

$$
\begin{aligned}
\left|F_{a b}\right| \cdot\left|e^{i \tau}-1\right|= & \left|e^{i\left(\omega_{a b}-\omega_{a b^{\prime}}\right)} F_{a b}-e^{i\left(\omega_{a^{\prime} b}-\omega_{a^{\prime} b^{\prime}}\right)} F_{a b}\right| \\
\leq & \left|e^{i\left(\omega_{a b}-\omega_{a b^{\prime}}\right)} F_{a b}-F_{a b^{\prime}}\right|+\left|F_{a b^{\prime}}-F_{a^{\prime} b^{\prime}}\right| \\
& +\left|e^{i\left(\omega_{a^{\prime} b}-\omega_{a^{\prime} b^{\prime}}\right)} F_{a^{\prime} b}-F_{a^{\prime} b^{\prime}}\right|+\left|F_{a b}-F_{a^{\prime} b}\right| \\
< & 4\left(C_{R}^{\prime}\right)^{2} L^{\delta+\delta^{\prime}} \sqrt{\varepsilon_{1} R}+8 \theta^{-1} L^{-2 / 3} \sqrt{R} .
\end{aligned}
$$

Comparing this with (3.30) and dividing by $\sqrt{R}$, we obtain

$$
\frac{C_{R}^{-\frac{2}{\delta}-\frac{2}{\delta^{\prime}}}}{8 \pi} L^{-1 / 3}<4\left(C_{R}^{\prime}\right)^{2} L^{\delta+\delta^{\prime}} \sqrt{\varepsilon_{1}}+8 \theta^{-1} L^{-2 / 3} .
$$

This gives a contradiction with the following consequences of (3.2) and (3.3):

$$
8 \theta^{-1} L^{-2 / 3} \leq \frac{C_{R}^{-\frac{2}{\delta}-\frac{2}{\delta^{\prime}}}}{16 \pi} L^{-1 / 3}, \quad 4\left(C_{R}^{\prime}\right)^{2} L^{\delta+\delta^{\prime}} \sqrt{\varepsilon_{1}} \leq \frac{C_{R}^{-\frac{2}{\delta}-\frac{2}{\delta^{\prime}}}}{16 \pi} L^{-1 / 3} .
$$

3.3. Proof of Theorem 1. We now show how to reduce Theorem 1 to Proposition 3.1. The idea is to split $G$ into pieces using a partition of unity. On each piece by appropriate rescaling we keep the regularity constant $C_{R}$ and reduce to the case (3.1) and $h=L^{-K}$ for some fixed $L$ satisfying (3.2) and some integer $K>0$.

To be more precise, let $\left(X, \mu_{X}\right),\left(Y, \mu_{Y}\right), \delta, \delta^{\prime}, I_{0}, J_{0}, \Phi, G$ satisfy the hypotheses of Theorem 1. Using a partition of unity, we write $G$ as a finite sum

$$
G=\sum_{\ell} G_{\ell}, \quad G_{\ell} \in C^{1}\left(I_{0} \times J_{0} ; \mathbb{C}\right), \quad \operatorname{supp} G_{\ell} \subset I_{\ell} \times J_{\ell}
$$

where $I_{\ell} \subset I_{0}, J_{\ell} \subset J_{0}$ are intervals such that for some $m=m(\ell) \in \mathbb{Z}$,

$$
2^{m-1}<\left|\partial_{x y}^{2} \Phi\right|<2^{m+1} \quad \text { on } I_{\ell} \times J_{\ell}
$$

It then suffices to show (1.4) where $G$ is replaced by one of the functions $G_{\ell}$. By changing $\Phi$ outside of the support of $G$ (which does not change the operator $\mathcal{B}_{h}$ ), we then reduce to the case when

$$
2^{m-1}<\left|\partial_{x y}^{2} \Phi\right|<2^{m+1} \quad \text { on } I_{0} \times J_{0}
$$

for some $m \in \mathbb{Z}$. 
We next rescale $\mathcal{B}_{h}$ to an operator $\widetilde{\mathcal{B}}_{\tilde{h}}$ satisfying the hypotheses of Proposition 3.1. Fix the smallest $L \in \mathbb{Z}$ satisfying (3.2). Choose $K \in \mathbb{Z}$ and $\sigma \in[1, \sqrt{L}$ ) such that

$$
\sigma^{2}=2^{m} \frac{\tilde{h}}{h}, \quad \tilde{h}:=L^{-K}
$$

Put for all intervals $I, J$

$$
\begin{gathered}
\widetilde{X}:=\sigma X \subset \tilde{I}_{0}:=\sigma I_{0}, \quad \widetilde{Y}:=\sigma Y \subset \tilde{J}_{0}:=\sigma J_{0}, \\
\mu_{\widetilde{X}}(\sigma I):=\sigma^{\delta} \mu_{X}(I), \quad \mu_{\widetilde{Y}}(\sigma J):=\sigma^{\delta^{\prime}} \mu_{Y}(J) .
\end{gathered}
$$

Then $\left(\tilde{X}, \mu_{\tilde{X}}\right)$ is $\delta$-regular, and $\left(\widetilde{Y}, \mu_{\tilde{Y}}\right)$ is $\delta^{\prime}$-regular, up to scale $\sigma h$ with regularity constant $C_{R}$. Consider the unitary operators

$$
\begin{array}{cc}
U_{X}: L^{2}\left(X, \mu_{X}\right) \rightarrow L^{2}\left(\tilde{X}, \mu_{\tilde{X}}\right), & U_{Y}: L^{2}\left(Y, \mu_{Y}\right) \rightarrow L^{2}\left(\tilde{Y}, \mu_{\tilde{Y}}\right), \\
U_{X} f(\tilde{x})=\sigma^{-\delta / 2} f\left(\sigma^{-1} \tilde{x}\right), & U_{Y} f(\tilde{y})=\sigma^{-\delta^{\prime} / 2} f\left(\sigma^{-1} \tilde{y}\right) .
\end{array}
$$

Then the operator $\widetilde{\mathcal{B}}_{\tilde{h}}:=U_{X} \mathcal{B}_{h} U_{Y}^{-1}: L^{2}\left(\widetilde{Y}, \mu_{\tilde{Y}}\right) \rightarrow L^{2}\left(\widetilde{X}, \mu_{\tilde{X}}\right)$ has the form (1.3):

$$
\widetilde{\mathcal{B}}_{\tilde{h}} f(\tilde{x})=\int_{\widetilde{Y}} \exp \left(\frac{i \widetilde{\Phi}(\tilde{x}, \tilde{y})}{\tilde{h}}\right) \widetilde{G}(\tilde{x}, \tilde{y}) f(\tilde{y}) d \mu_{\tilde{Y}}(\tilde{y})
$$

where

$$
\widetilde{\Phi}(\tilde{x}, \tilde{y})=2^{-m} \sigma^{2} \Phi\left(\sigma^{-1} \tilde{x}, \sigma^{-1} \tilde{y}\right), \quad \widetilde{G}(\tilde{x}, \tilde{y})=\sigma^{-\frac{\delta}{2}-\frac{\delta^{\prime}}{2}} G\left(\sigma^{-1} \tilde{x}, \sigma^{-1} \tilde{y}\right) .
$$

By (3.32) the function $\widetilde{\Phi}$ satisfies (3.1). Fix smallest $K_{0} \in \mathbb{N}_{0}$ such that $\sigma h \leq L^{K_{0}-K}$, that is

$$
L^{K_{0}} \geq \frac{2^{m}}{\sigma}
$$

Without loss of generality, we may assume that $h$ is small enough depending on $L, m$ so that $K \geq 2 K_{0}$. Then Proposition 3.1 applies to $\widetilde{\mathcal{B}}_{\tilde{h}}$ and gives

$$
\left\|\mathcal{B}_{h}\right\|_{L^{2}\left(Y, \mu_{Y}\right) \rightarrow L^{2}\left(X, \mu_{X}\right)}=\left\|\widetilde{\mathcal{B}}_{\tilde{h}}\right\|_{L^{2}\left(\widetilde{Y}, \mu_{\tilde{Y}}\right) \rightarrow L^{2}\left(\widetilde{X}, \mu_{\tilde{X}}\right)} \leq C \tilde{h}^{\varepsilon_{0}} \leq C\left(2^{-m} L\right)^{\varepsilon_{0}} h^{\varepsilon_{0}}
$$

for $\varepsilon_{0}$ defined in (1.5) and some constant $C$ depending only on $\delta, \delta^{\prime}, C_{R}, I_{0}, J_{0}, \Phi, G$. This finishes the proof of Theorem 1 .

\section{Application: SPeCtral Gap FOR hyperbolic SURFACES}

We now discuss applications of Theorem 1 to spectral gaps. We start with the case of hyperbolic surfaces, referring the reader to the book of Borthwick [Bo16] and to [DyZa16] for the terminology used here.

Let $M=\Gamma \backslash \mathbb{H}^{2}$ be a convex co-compact hyperbolic surface, $\Lambda_{\Gamma} \subset \mathbb{S}^{1}$ be its limit set, $\delta \in[0,1)$ be the dimension of $\Lambda_{\Gamma}$, and $\mu$ be the Patterson-Sullivan measure, which is a probability measure supported on $\Lambda_{\Gamma}$, see for instance [Bo16, $\left.\S 14.1\right]$. Since $\Lambda_{\Gamma}$ is closed and is not equal to the entire $\mathbb{S}^{1}$, we may cut the circle $\mathbb{S}^{1}$ to turn it into an interval and treat $\Lambda_{\Gamma}$ as a compact subset of $\mathbb{R}$. Then $\left(\Lambda_{\Gamma}, \mu\right)$ is $\delta$-regular up to scale 0 with 
some constant $C_{R}$, see for instance [Bo16, Lemma 14.13]. The regularity constant $C_{R}$ depends continuously on the surface, as explained in the case of three-funnel surfaces in [DyZa16, Proposition 7.7].

The main result of this section is the following essential spectral gap for $M$. We formulate it here in terms of scattering resolvent of the Laplacian. Another formulation is in terms of a zero free region for the Selberg zeta function past the first pole, see for instance [DyZa16]. See below for a discussion of previous work on spectral gaps.

Theorem 2. Consider the meromorphic scattering resolvent

$$
R(\lambda)=\left(-\Delta_{M}-\frac{1}{4}-\lambda^{2}\right)^{-1}:\left\{\begin{array}{l}
L^{2}(M) \rightarrow L^{2}(M), \quad \operatorname{Im} \lambda>0, \\
L_{\text {comp }}^{2}(M) \rightarrow L_{\operatorname{loc}}^{2}(M), \quad \operatorname{Im} \lambda \leq 0 .
\end{array}\right.
$$

Assume that $0<\delta<1$. Then $M$ has an essential spectral gap of size

$$
\beta=\frac{1}{2}-\delta+\left(13 C_{R}\right)^{-\frac{320}{\delta(1-\delta)}}
$$

that is $R(\lambda)$ has only finitely many poles in $\{\operatorname{Im} \lambda>-\beta\}$ and it satisfies the cutoff estimates for each $\psi \in C_{0}^{\infty}(M), \varepsilon>0$ and some constant $C_{0}$ depending on $\varepsilon$

$$
\|\psi R(\lambda) \psi\|_{L^{2} \rightarrow L^{2}} \leq C(\psi, \varepsilon)|\lambda|^{-1-2 \min (0, \operatorname{Im} \lambda)+\varepsilon}, \quad \operatorname{Im} \lambda \in[-\beta, 1], \quad|\operatorname{Re} \lambda| \geq C_{0} .
$$

Proof. We use the strategy of [DyZa16]. By [DyZa16, Theorem 3], it suffices to show the following fractal uncertainty principle: for each $\rho \in(0,1)$,

$$
\beta_{0}:=\frac{1}{2}-\delta+\left(150 C_{R}^{2}\right)^{-\frac{160}{\delta(1-\delta)}}
$$

and each cutoff function $\chi \in C^{\infty}\left(\mathbb{S}^{1} \times \mathbb{S}^{1}\right)$ supported away from the diagonal, there exists a constant $C$ depending on $M, \chi, \rho$ such that for all $h \in(0,1)$

$$
\left\|\mathbb{1}_{\Lambda_{\Gamma}\left(h^{\rho}\right)} B_{\chi, h} \mathbb{1}_{\Lambda_{\Gamma}\left(h^{\rho}\right)}\right\|_{L^{2}\left(\mathbb{S}^{1}\right) \rightarrow L^{2}\left(\mathbb{S}^{1}\right)} \leq C h^{\beta_{0}-2(1-\rho)}
$$

where $\Lambda_{\Gamma}\left(h^{\rho}\right) \subset \mathbb{S}^{1}$ is the $h^{\rho}$ neighborhood of $\Lambda_{\Gamma}$ and the operator $B_{\chi, h}$ is defined by (here $|x-y|$ is the Euclidean distance between $x, y \in \mathbb{S}^{1} \subset \mathbb{R}^{2}$ )

$$
B_{\chi, h} f(x)=(2 \pi h)^{-1 / 2} \int_{\mathbb{S}^{1}}|x-y|^{2 i / h} \chi(x, y) f(y) d y .
$$

To show (4.2), we first note that by Lemma 2.2, $\left(Y, \mu_{Y}\right)$ is $\delta$-regular up to scale $h$ with constant $30 C_{R}^{2}$, where $Y=\Lambda_{\Gamma}(h)$ and $\mu_{Y}$ is $h^{\delta-1}$ times the restriction of the Lebesgue measure to $Y$. We lift $\chi(x, y)$ to a compactly supported function on $\mathbb{R}^{2}$ (splitting it into pieces using a partition of unity) and write

$$
B_{\chi, h} \mathbb{1}_{\Lambda_{\Gamma}(h)} f(x)=(2 \pi)^{-1 / 2} h^{1 / 2-\delta} \mathcal{B}_{h} f(x),
$$

where $\mathcal{B}_{h}$ has the form (1.3) with $G(x, y)=\chi(x, y)$ and (with $|x-y|$ still denoting the Euclidean distance between $x, y \in \mathbb{S}^{1}$ )

$$
\Phi(x, y)=2 \log |x-y| .
$$


The function $\Phi$ is smooth and satisfies the condition $\partial_{x y}^{2} \Phi \neq 0$ on the open set $\mathbb{S}^{1} \times \mathbb{S}^{1} \backslash$ $\{x=y\}$ which contains the support of $G$, see for instance [BoDy16, §4.3]. Applying Theorem 1 with $\left(X, \mu_{X}\right):=\left(Y, \mu_{Y}\right)$, we obtain

$$
\left\|\mathbb{1}_{\Lambda_{\Gamma}(h)} B_{\chi, h} \mathbb{1}_{\Lambda_{\Gamma}(h)}\right\|_{L^{2}\left(\mathbb{S}^{1}\right) \rightarrow L^{2}\left(\mathbb{S}^{1}\right)} \leq C h^{\beta_{0}} .
$$

Similarly we have

$$
\left\|\mathbb{1}_{\Lambda_{\Gamma}(h)+t} B_{\chi, h} \mathbb{1}_{\Lambda_{\Gamma}(h)+s}\right\|_{L^{2}\left(\mathbb{S}^{1}\right) \rightarrow L^{2}\left(\mathbb{S}^{1}\right)} \leq C h^{\beta_{0}}, \quad t, s \in[-1,1]
$$

where $X+t$ is the result of rotating $X \subset \mathbb{S}^{1}$ by angle $t$. Covering $\Lambda_{\Gamma}\left(h^{\rho}\right)$ with at most $10 h^{\rho-1}$ rotations of the set $\Lambda_{\Gamma}(h)$ (see for instance the proof of [BoDy16, Proposition 4.2]) and using triangle inequality, we obtain (4.2), finishing the proof.

We now briefly discuss previous results on spectral gaps for hyperbolic surfaces:

- The works of Patterson [Pa76] and Sullivan [Su79] imply that $R(\lambda)$ has no poles with $\operatorname{Im} \lambda>\delta-\frac{1}{2}$. On the other hand, the fact that $R(\lambda)$ is the $L^{2}$ resolvent of the Laplacian in $\{\operatorname{Im} \lambda>0\}$ shows that it has only has finitely many poles in this region. Together these two results give the essential spectral gap $\beta=\max \left(0, \frac{1}{2}-\delta\right)$. Thus Theorem 2 gives no new results when $\delta$ is much larger than $\frac{1}{2}$.

- Using the method developed by Dolgopyat [Do98], Naud [Na05] showed an essential spectral gap of size $\beta>\frac{1}{2}-\delta$ when $\delta>0$. Oh-Winter [OhWi16] showed that the size of the gap is uniformly controlled for towers of congruence covers in the arithmetic case.

- Dyatlov-Zahl [DyZa16] introduced the fractal uncertainty principle approach to spectral gaps and used it together with tools from additive combinatorics to give an estimate of the size of the gap in terms of $C_{R}$ in the case when $\delta$ is very close to $\frac{1}{2}$.

- Bourgain-Dyatlov [BoDy16] showed that each convex co-compact hyperbolic surface has an essential spectral gap of some size $\beta=\beta\left(\delta, C_{R}\right)>0$. Their result is new in the case $\delta>\frac{1}{2}$ and is thus complementary to the results mentioned above as well as to Theorem 2 .

More generally, spectral gaps have been studied for noncompact manifolds with hyperbolic trapped sets. (See for instance [No11, §2.1] for a definition.) In this setting the Patterson-Sullivan gap $\frac{1}{2}-\delta$ generalizes to the pressure gap $-P\left(\frac{1}{2}\right)$ which has been established by Ikawa [Ik88], Gaspard-Rice [GaRi89], and NonnenmacherZworski [NoZw09]. An improved gap $\beta>-P\left(\frac{1}{2}\right)$ has been proved in several cases, see in particular Petkov-Stoyanov [PeSt10] and Stoyanov [St11, St12]. We refer the reader to the review of Nonnenmacher [No11] for an overview of results on spectral gaps for general hyperbolic trapped sets. 


\section{ApPlicAtion: SPECTRAL GAP FOR OPEN QUANTUM MAPS}

In this section, we discuss applications of fractal uncertainty principle to the spectral properties of open quantum maps. Following the notation in [DyJi17] we consider an open quantum baker's map $B_{N}$ determined by a triple $(M, \mathcal{A}, \chi)$ where $M \in \mathbb{N}$ is called the base, $\mathcal{A} \subset \mathbb{Z}_{M}=\{0,1, \ldots, M-1\}$ is called the alphabet, and $\chi \in C_{0}^{\infty}((0,1) ;[0,1])$ is a cutoff function. The map $B_{N}$ is a sequence of operators $B_{N}: \ell_{N}^{2} \rightarrow \ell_{N}^{2}, \ell_{N}^{2}=$ $\ell^{2}\left(\mathbb{Z}_{N}\right)$, defined for every positive $N \in M \mathbb{Z}$ by

$$
B_{N}=\mathcal{F}_{N}^{*}\left(\begin{array}{lll}
\chi_{N / M} \mathcal{F}_{N / M} \chi_{N / M} & & \\
& \ddots & \\
& & \chi_{N / M} \mathcal{F}_{N / M} \chi_{N / M}
\end{array}\right) I_{\mathcal{A}, M}
$$

where $\mathcal{F}_{N}$ is the unitary Fourier transform given by the $N \times N$ matrix $\frac{1}{\sqrt{N}}\left(e^{-2 \pi i j \ell / N}\right)_{j \ell}$, $\chi_{N / M}$ is the multiplication operator on $\ell_{N / M}^{2}$ discretizing $\chi$, and $I_{\mathcal{A}, M}$ is the diagonal matrix with $\ell$-th diagonal entry equal to 1 if $\left\lfloor\frac{\ell}{N / M}\right\rfloor \in \mathcal{A}$ and 0 otherwise.

An important difference from [DyJi17] is that in the present paper we allow $N$ to be any multiple of $M$, while [DyJi17] required that $N$ be a power of $M$. To measure the size of $N$, we let $k$ be the unique integer such that $M^{k} \leq N<M^{k+1}$, i.e. $k=\left\lfloor\frac{\log N}{\log M}\right\rfloor$. Denote by $\delta$ the dimension of the Cantor set corresponding to $M$ and $\mathcal{A}$, given by

$$
\delta=\frac{\log |\mathcal{A}|}{\log M}
$$

The main result of this section is the following spectral gap, which was previously established in [DyJi17, Theorem 1] for the case when $N$ is a power of $M$ :

Theorem 3. Assume that $0<\delta<1$, that is $1<|\mathcal{A}|<M$. Then there exists

$$
\beta=\beta(M, \mathcal{A})>\max \left(0, \frac{1}{2}-\delta\right)
$$

such that, with $\operatorname{Sp}\left(B_{N}\right) \subset\{\lambda \in \mathbb{C}:|\lambda| \leq 1\}$ denoting the spectrum of $B_{N}$,

$$
\limsup _{N \rightarrow \infty, N \in M \mathbb{Z}} \max \left\{|\lambda|: \lambda \in \operatorname{Sp}\left(B_{N}\right)\right\} \leq M^{-\beta} .
$$

The main component of the proof is a fractal uncertainty principle. For the case $N=M^{k}$, the following version of it was used in [DyJi17]:

$$
\left\|\mathbb{1}_{\mathcal{C}_{k}} \mathcal{F}_{N} \mathbb{1}_{\mathcal{C}_{k}}\right\|_{\ell_{N}^{2} \rightarrow \ell_{N}^{2}} \leq C N^{-\beta}
$$

where $\mathcal{C}_{k}$ is the discrete Cantor set given by

$$
\mathcal{C}_{k}:=\left\{\sum_{j=0}^{k-1} a_{j} M^{j} \mid a_{0}, \ldots, a_{k-1} \in \mathcal{A}\right\} \subset \mathbb{Z}_{N} .
$$


For general $N \in M \mathbb{Z} \cap\left[M^{k}, M^{k+1}\right)$, we define a similar discrete Cantor set in $\mathbb{Z}_{N}$ by

$$
\mathcal{C}_{k}(N):=\left\{b_{j}(N): j \in \mathcal{C}_{k}\right\} \subset \mathbb{Z}_{N}, \quad b_{j}(N):=\left\lceil\frac{j N}{M^{k}}\right\rceil .
$$

In fact, in our argument we only need $b_{j}(N)$ to be some integer in $\left[\frac{j N}{M^{k}}, \frac{(j+1) N}{M^{k}}\right)$.

The uncertainty principle then takes the following form:

Theorem 4. Assume that $0<\delta<1$. Then there exists

$$
\beta=\beta(M, \mathcal{A})>\max \left(0, \frac{1}{2}-\delta\right)
$$

such that for some constant $C$ and all $N$,

$$
\left\|\mathbb{1}_{\mathcal{C}_{k}(N)} \mathcal{F}_{N} \mathbb{1}_{\mathcal{C}_{k}(N)}\right\|_{\ell_{N}^{2} \rightarrow \ell_{N}^{2}} \leq C N^{-\beta}
$$

In $\$ 5.1$ below, we show that Theorem 4 implies Theorem 3 . We prove Theorem 4 in $§ \S 5.3,5.4$, using Ahlfors-David regularity of the Cantor set which is verified in $§ 5.2$.

5.1. Fractal uncertainty principle implies spectral gap. We first show that Theorem 4 implies Theorem 3. The argument is essentially the same as in [DyJi17, Section 2.3], relying on the following generalization of [DyJi17, Proposition 2.5]:

Proposition 5.1 (Localization of eigenstates). Fix $\nu>0, \rho \in(0,1)$, and assume that for some $k \in \mathbb{N}, N \in M \mathbb{Z} \cap\left[M^{k}, M^{k+1}\right), \lambda \in \mathbb{C}, u \in \ell_{N}^{2}$, we have

$$
B_{N} u=\lambda u, \quad|\lambda| \geq M^{-\nu} .
$$

Define

$$
X_{\rho}:=\bigcup\left\{\mathcal{C}_{k}(N)+m: m \in \mathbb{Z},|m| \leq(M+2) N^{1-\rho}\right\} \subset \mathbb{Z}_{N}
$$

Then

$$
\begin{gathered}
\|u\|_{\ell_{N}^{2}} \leq M^{\nu}|\lambda|^{-\rho k}\left\|\mathbb{1}_{X_{\rho}} u\right\|_{\ell_{N}^{2}}+\mathcal{O}\left(N^{-\infty}\right)\|u\|_{\ell_{N}^{2}}, \\
\left\|u-\mathcal{F}_{N}^{*} \mathbb{1}_{X_{\rho}} \mathcal{F}_{N} u\right\|_{\ell_{N}^{2}}=\mathcal{O}\left(N^{-\infty}\right)\|u\|_{\ell_{N}^{2}}
\end{gathered}
$$

where the constants in $\mathcal{O}\left(N^{-\infty}\right)$ depend only on $\nu, \rho, \chi$.

Proof. Following [DyJi17, (2.7)], let $\Phi=\Phi_{M, \mathcal{A}}$ be the expanding map defined by

$$
\Phi: \bigsqcup_{a \in \mathcal{A}}\left(\frac{a}{M}, \frac{a+1}{M}\right) \rightarrow(0,1) ; \quad \Phi(x)=M x-a, \quad x \in\left(\frac{a}{M}, \frac{a+1}{M}\right) .
$$

Put

$$
\tilde{k}:=\lceil\rho k\rceil \in\{1, \ldots, k\} .
$$

With $d(\cdot, \cdot)$ denoting the distance function on the circle as in [DyJi17, §2.1], define

$$
\mathcal{X}_{\rho}:=\left\{x \in[0,1]: d\left(x, \Phi^{-\tilde{k}}([0,1])\right) \leq N^{-\rho}\right\} .
$$


Then (5.9), (5.10) follow from the long time Egorov theorem [DyJi17, Proposition 2.4] (whose proof never used that $N$ is a power of $M$ ) similarly to [DyJi17, Proposition 2.5], as long as we show the following analog of [DyJi17, (2.30)]:

$$
\ell \in\{0, \ldots, N-1\}, \quad \frac{\ell}{N} \in \mathcal{X}_{\rho} \Longrightarrow \ell \in X_{\rho} .
$$

To see (5.13), note that (with the intervals considered in $\mathbb{R} / \mathbb{Z}$ )

$$
\Phi^{-\tilde{k}}([0,1]) \subset \bigcup_{j \in \mathcal{C}_{k}}\left(\frac{j-M^{k-\tilde{k}}}{M^{k}}, \frac{j+M^{k-\tilde{k}}}{M^{k}}\right) .
$$

Assume that $\ell \in\{0, \ldots, N-1\}$ and $\ell / N \in \mathcal{X}_{\rho}$. Then there exists $j \in \mathcal{C}_{k}$ such that

$$
d\left(\frac{\ell}{N}, \frac{j}{M^{k}}\right) \leq N^{-\rho}+M^{-\tilde{k}} \leq(M+1) N^{-\rho} .
$$

It follows that

$$
d\left(\frac{\ell}{N}, \frac{b_{j}(N)}{N}\right) \leq(M+2) N^{-\rho}
$$

and thus $\ell \in X_{\rho}$ as required.

Now, we assume that Theorem 4 holds and prove Theorem 3. Using the triangle inequality as in the proof of [DyJi17, Proposition 2.6], we obtain

$$
\begin{aligned}
\left\|\mathbb{1}_{X_{\rho}} \mathcal{F}_{N}^{*} \mathbb{1}_{X_{\rho}}\right\|_{\ell_{N}^{2} \rightarrow \ell_{N}^{2}} & \leq(2 M+5)^{2} N^{2(1-\rho)}\left\|\mathbb{1}_{\mathcal{C}_{k}(N)} \mathcal{F}_{N} \mathbb{1}_{\mathcal{C}_{k}(N)}\right\|_{\ell_{N}^{2} \rightarrow \ell_{N}^{2}} \\
& \leq C N^{2(1-\rho)-\beta} .
\end{aligned}
$$

Here $C$ denotes a constant independent of $N$.

Assume that $\lambda \in \mathbb{C}$ is an eigenvalue of $B_{N}$ such that $|\lambda| \geq M^{-\beta}$ and $u \in \ell_{N}^{2}$ is a normalized eigenfunction of $B_{N}$ with eigenvalue $\lambda$. By (5.9), (5.10), and (5.14)

$$
\begin{aligned}
1=\|u\|_{\ell_{N}^{2}} & \leq M^{\beta}|\lambda|^{-\rho k}\left\|\mathbb{1}_{X_{\rho}} u\right\|_{\ell_{N}^{2}}+\mathcal{O}\left(N^{-\infty}\right) \\
& \leq M^{\beta}|\lambda|^{-\rho k}\left\|\mathbb{1}_{X_{\rho}} \mathcal{F}_{N}^{*} \mathbb{1}_{X_{\rho}} \mathcal{F}_{N} u\right\|_{\ell_{N}^{2}}+\mathcal{O}\left(N^{-\infty}\right) \\
& \leq C|\lambda|^{-\rho k} N^{2(1-\rho)-\beta}+\mathcal{O}\left(N^{-\infty}\right) .
\end{aligned}
$$

It follows that $|\lambda|^{\rho k} \leq C N^{-\beta+2(1-\rho)}$ or equivalently

$$
|\lambda| \leq C^{1 / \rho k} M^{(2(1-\rho)-\beta) / \rho} .
$$

This implies that

$$
\limsup _{N \rightarrow \infty} \max \left\{|\lambda|: \lambda \in \operatorname{Sp}\left(B_{N}\right)\right\} \leq \max \left\{M^{-\beta}, M^{(2(1-\rho)-\beta) / \rho}\right\} .
$$

Letting $\rho \rightarrow 1$, we conclude the proof of Theorem 3 . 
5.2. Regularity of discrete Cantor sets. Theorem 4 will be deduced from Theorem 1 and the results of [BoDy16]. To apply these, we establish Ahlfors-David regularity of the Cantor set $\mathcal{C}_{k}(N) \subset \mathbb{Z}_{N}=\{0, \ldots, N-1\}$ in the following discrete sense.

Definition 5.2. We say that $X \subset \mathbb{Z}_{N}$ is $\delta$-regular with constant $C_{R}$ if

- for each interval $J$ of size $|J| \geq 1$, we have $\#(J \cap X) \leq C_{R}|J|^{\delta}$, and

- for each interval $J$ with $1 \leq|J| \leq N$ which is centered at a point in $X$, we have $\#(J \cap X) \geq C_{R}^{-1}|J|^{\delta}$.

Definition 5.2 is related to Definition 1.1 as follows:

Lemma 5.3. Let $X \subset \mathbb{Z}_{N}$. Define $\widetilde{X}:=N^{-1} X \subset[0,1]$ which supports the measure

$$
\mu_{\tilde{X}}(A):=N^{-\delta} \cdot \#(\tilde{X} \cap A), \quad A \subset \mathbb{R} .
$$

Then $X$ is $\delta$-regular with constant $C_{R}$ in the sense of Definition 5.2 if and only if $\left(\widetilde{X}, \mu_{\tilde{X}}\right)$ is $\delta$-regular up to scale $N^{-1}$ with constant $C_{R}$ in the sense of Definition 1.1 .

Proof. This follows directly from the two definitions.

We first establish the regularity of the discrete Cantor set $\mathcal{C}_{k}$ defined in (5.5):

Lemma 5.4. The set $\mathcal{C}_{k} \subset \mathbb{Z}_{M^{k}}$ is $\delta$-regular with constant $C_{R}=2 M^{2 \delta}$.

Proof. We notice that for all integers $k^{\prime} \in[0, k]$ and $j^{\prime} \in \mathbb{Z}$

$$
\#\left(\mathcal{C}_{k} \cap\left[j^{\prime} M^{k^{\prime}},\left(j^{\prime}+1\right) M^{k^{\prime}}\right)\right)= \begin{cases}|\mathcal{A}|^{k^{\prime}}=M^{\delta k^{\prime}}, & j^{\prime} \in \mathcal{C}_{k-k^{\prime}} \\ 0, & j^{\prime} \notin \mathcal{C}_{k-k^{\prime}}\end{cases}
$$

Let $J$ be an interval in $\mathbb{R}$, with $1 \leq|J| \leq N=M^{k}$. Choose an integer $k^{\prime} \in[0, k-1]$ such that $M^{k^{\prime}} \leq|J| \leq M^{k^{\prime}+1}$. Then there exists some $j^{\prime} \in \mathbb{Z}$ such that

$$
J \subset\left[j^{\prime} M^{k^{\prime}+1},\left(j^{\prime}+2\right) M^{k^{\prime}+1}\right) .
$$

Therefore by (5.17)

$$
\#\left(\mathcal{C}_{k} \cap J\right) \leq 2 M^{\delta\left(k^{\prime}+1\right)} \leq 2 M^{\delta}|J|^{\delta} \leq C_{R}|J|^{\delta} .
$$

On the other hand, if $|J|>N$ then

$$
\#\left(\mathcal{C}_{k} \cap J\right) \leq \#\left(\mathcal{C}_{k}\right)=N^{\delta} \leq|J|^{\delta} .
$$

This gives the required upper bound on $\#\left(\mathcal{C}_{k} \cap J\right)$.

Now, assume that $1 \leq|J| \leq N$ and $J$ is centered at some $j \in \mathcal{C}_{k}$. Choose $k^{\prime}$ as before. If $k^{\prime}=0$ then

$$
\#\left(\mathcal{C}_{k} \cap J\right) \geq 1 \geq M^{-\delta}|J|^{\delta} \geq C_{R}^{-1}|J|^{\delta} .
$$


We henceforth assume that $1 \leq k^{\prime} \leq k-1$. Let $j^{\prime} \in \mathcal{C}_{k-k^{\prime}+1}$ be the unique element such that $j^{\prime} M^{k^{\prime}-1} \leq j<\left(j^{\prime}+1\right) M^{k^{\prime}-1}$. Since $M \geq 2$, we have $|J| \geq M^{k^{\prime}} \geq 2 M^{k^{\prime}-1}$ and thus

$$
\left[j^{\prime} M^{k^{\prime}-1},\left(j^{\prime}+1\right) M^{k^{\prime}-1}\right] \subset\left[j-M^{k^{\prime}-1}, j+M^{k^{\prime}-1}\right] \subset J .
$$

Therefore by (5.17)

$$
\#\left(\mathcal{C}_{k} \cap J\right) \geq M^{\delta\left(k^{\prime}-1\right)} \geq M^{-2 \delta}|J|^{\delta} \geq C_{R}^{-1}|J|^{\delta} .
$$

This gives the required lower bound on $\#\left(\mathcal{C}_{k} \cap J\right)$, finishing the proof.

We now establish regularity of the dilated Cantor set $\mathcal{C}_{k}(N)$ :

Proposition 5.5. Assume that $M^{k} \leq N<M^{k+1}$ and let $\mathcal{C}_{k}(N) \subset \mathbb{Z}_{N}$ be given by (5.6). Then $\mathcal{C}_{k}(N)$ is $\delta$-regular with constant $C_{R}=8 M^{3 \delta}$.

Proof. For any interval $J$, we have

$$
\#\left(\mathcal{C}_{k}(N) \cap J\right)=\#\left\{j \in \mathcal{C}_{k}: b_{j}(N) \in J\right\}=\#\left\{j \in \mathcal{C}_{k}: \frac{M^{k}}{N} b_{j}(N) \in \frac{M^{k}}{N} J\right\} .
$$

By our choice of $b_{j}(N)$, we have $\frac{M^{k}}{N} b_{j}(N) \in[j, j+1)$. Therefore

$$
\#\left(\mathcal{C}_{k} \cap \frac{M^{k}}{N} J\right)-1 \leq \#\left(\mathcal{C}_{k}(N) \cap J\right) \leq \#\left(\mathcal{C}_{k} \cap \frac{M^{k}}{N} J\right)+1 .
$$

We apply Lemma 5.4 to see that for any interval $J$ with $|J| \geq 1$

$$
\#\left(\mathcal{C}_{k}(N) \cap J\right) \leq 2 M^{2 \delta}|J|^{\delta}+1 \leq 3 M^{2 \delta}|J|^{\delta} \leq C_{R}|J|^{\delta} .
$$

Now, assume that $J$ is an interval with $8^{1 / \delta} M^{3} \leq|J| \leq N$ centered at $b_{j}(N)$ for some $j \in \mathcal{C}_{k}$. Then $\frac{M^{k}}{N} J$ contains the interval of size $\frac{1}{2 M}|J|$ centered at $j$. Therefore, by Lemma 5.4

$$
\#\left(\mathcal{C}_{k}(N) \cap J\right) \geq \frac{1}{2 M^{2 \delta}}\left(\frac{|J|}{2 M}\right)^{\delta}-1 \geq \frac{|J|^{\delta}}{8 M^{3 \delta}} \geq C_{R}^{-1}|J|^{\delta} .
$$

Finally, if $J$ is an interval with $1 \leq|J| \leq 8^{1 / \delta} M^{3}$ centered at a point in $\mathcal{C}_{k}(N)$, then

$$
\#\left(\mathcal{C}_{k}(N) \cap J\right) \geq 1 \geq C_{R}^{-1}|J|^{\delta} .
$$

5.3. Fractal uncertainty principle for $\delta \leq 1 / 2$. The proof of Theorem 4 in the case $\delta \leq 1 / 2$ relies on the following corollary of Theorem 1 :

Proposition 5.6. Let $X, Y \subset \mathbb{Z}_{N}$ be $\delta$-regular with constant $C_{R}$ and $0<\delta<1$. Then

$$
\left\|\mathbb{1}_{X} \mathcal{F}_{N} \mathbb{1}_{Y}\right\|_{\ell_{N}^{2} \rightarrow \ell_{N}^{2}} \leq C N^{-\left(\frac{1}{2}-\delta+\varepsilon_{0}\right)}
$$

where $C$ only depends on $\delta, C_{R}$ and

$$
\varepsilon_{0}=\left(5 C_{R}\right)^{-\frac{160}{\delta(1-\delta)}} .
$$


Proof. Put $h:=N^{-1}, \widetilde{X}:=h X, \widetilde{Y}:=h Y$, and define the measures $\mu_{\widetilde{X}}, \mu_{\widetilde{Y}}$ by $(5.16)$. By Lemma 5.3, $\left(\widetilde{X}, \mu_{\tilde{X}}\right)$ and $\left(\widetilde{Y}, \mu_{\tilde{Y}}\right)$ are $\delta$-regular up to scale $h$ with constant $C_{R}$. Consider the operator $\mathcal{B}_{h}: L^{1}\left(\widetilde{Y}, \mu_{\widetilde{Y}}\right) \rightarrow L^{\infty}\left(\widetilde{X}, \mu_{\widetilde{X}}\right)$ defined by

$$
\mathcal{B}_{h} f(x)=\int_{\widetilde{Y}} \exp \left(-\frac{2 \pi i x y}{h}\right) f(y) d \mu_{\widetilde{Y}}(y)
$$

and note that it has the form (1.3) with $\Phi(x, y)=-2 \pi x y, G \equiv 1$. By Theorem 1

$$
\left\|\mathcal{B}_{h}\right\|_{L^{2}\left(\widetilde{Y}, \mu_{\widetilde{Y}}\right) \rightarrow L^{2}\left(\widetilde{X}, \mu_{\widetilde{X}}\right)} \leq C h^{\varepsilon_{0}} .
$$

Comparing the formula

$$
\mathcal{B}_{h} f\left(\frac{j}{N}\right)=N^{-\delta} \sum_{\ell \in Y} \exp \left(-\frac{2 \pi i j \ell}{N}\right) f\left(\frac{\ell}{N}\right), \quad j \in X
$$

with the definition of the discrete Fourier transform $\mathcal{F}_{N}$, we see that

$$
\left\|\mathbb{1}_{X} \mathcal{F}_{N} \mathbb{1}_{Y}\right\|_{\ell_{N}^{2} \rightarrow \ell_{N}^{2}}=N^{\delta-1 / 2}\left\|\mathcal{B}_{h}\right\|_{L^{2}\left(\widetilde{Y}, \mu_{\tilde{Y}}\right) \rightarrow L^{2}\left(\widetilde{X}, \mu_{\tilde{X}}\right)}
$$

which finishes the proof.

Combining Propositions 5.5 and 5.6, we get (5.8) for

$$
\beta=\frac{1}{2}-\delta+\left(40 M^{3 \delta}\right)^{-\frac{160}{\delta(1-\delta)}}
$$

which finishes the proof of Theorem 4 for $\delta \leq 1 / 2$.

5.4. Fractal uncertainty principle for $\delta>1 / 2$. For $\delta>1 / 2$, Theorem 1 does not in general give an improvement over the trivial gap $\beta=0$. Instead, we shall use the following reformulation of [BoDy16, Theorem 4]:

Proposition 5.7. Let $0 \leq \delta<1, C_{R} \geq 1, N \geq 1$ and assume that $\widetilde{X}, \widetilde{Y} \subset[-1,1]$ and $\left(\widetilde{X}, \mu_{\tilde{X}}\right)$ and $\left(\widetilde{Y}, \mu_{\widetilde{Y}}\right)$ are $\delta$-regular up to scale $N^{-1}$ with constant $C_{R}$ in the sense of Definition 1.1, for some finite measures $\mu_{\widetilde{X}}, \mu_{\widetilde{Y}}$ supported on $\widetilde{X}, \widetilde{Y}$.

Then there exist $\beta_{0}>0, C_{0}$ depending only on $\delta, C_{R}$ such that for all $f \in L^{2}(\mathbb{R})$,

$$
\operatorname{supp} \hat{f} \subset N \cdot \tilde{Y} \quad \Longrightarrow \quad\|f\|_{L^{2}(\widetilde{X})} \leq C_{0} N^{-\beta_{0}}\|f\|_{L^{2}(\mathbb{R})} \text {. }
$$

Here $\hat{f}$ denotes the Fourier transform of $f$ :

$$
\hat{f}(\xi)=\mathcal{F} f(\xi)=\int_{\mathbb{R}} e^{-2 \pi i x \xi} f(x) d x .
$$

Proposition 5.7 implies the following discrete fractal uncertainty principle:

Proposition 5.8. Let $X, Y \subset \mathbb{Z}_{N}$ be $\delta$-regular with constant $C_{R}$ and $0 \leq \delta<1$. Then

$$
\left\|\mathbb{1}_{X} \mathcal{F}_{N} \mathbb{1}_{Y}\right\|_{\ell_{N}^{2} \rightarrow \ell_{N}^{2}} \leq C N^{-\beta}
$$

where $C, \beta>0$ only depend on $\delta, C_{R}$. 
Proof. Put $h:=N^{-1}$,

$$
\widetilde{X}:=h X+[-h, h], \quad \widetilde{Y}:=h Y+[-h, h],
$$

and define the measures $\mu_{\tilde{X}}, \mu_{\tilde{Y}}$ on $\tilde{X}, \tilde{Y}$ by (2.6). By Lemmas 5.3 and $2.2,\left(\tilde{X}, \mu_{\tilde{X}}\right)$ and $\left(\tilde{Y}, \mu_{\tilde{Y}}\right)$ are $\delta$-regular up to scale $h$ with constant $30 C_{R}^{2}$. Applying Proposition 5.7, we obtain for some constants $\beta_{0}>0, C_{0}$ depending only on $\delta, C_{R}$ and all $f \in L^{2}(\mathbb{R})$

$$
\operatorname{supp} \hat{f} \subset N \cdot \tilde{Y} \quad \Longrightarrow \quad\|f\|_{L^{2}(\tilde{X})} \leq C_{0} N^{-\beta_{0}}\|f\|_{L^{2}(\mathbb{R})}
$$

To pass from (5.24) to (5.23), fix a cutoff function $\chi$ such that for some constant $c>0$

$$
\chi \in C_{0}^{\infty}((-1 / 2,1 / 2)), \quad\|\chi\|_{L^{2}}=1, \quad \inf _{[0,1]}\left|\mathcal{F}^{-1} \chi\right| \geq c
$$

This is possible since for any $\chi \in C_{0}^{\infty}(\mathbb{R})$ which is not identically $0, \mathcal{F}^{-1} \chi$ extends to an entire function and thus has no zeroes on $\{\operatorname{Im} z=s\}$ for all but countably many choices of $s \in \mathbb{R}$. Choosing such $s$ we see that $\mathcal{F}^{-1}\left(e^{-s \xi} \chi(\xi)\right)$ has no real zeroes.

Now, take arbitrary $u \in \ell_{N}^{2}$. Consider the function $f \in L^{2}(\mathbb{R})$ defined by

$$
\hat{f}(\xi)=\sum_{\ell \in Y} u(\ell) \chi(\xi-\ell)
$$

Then $\operatorname{supp} \hat{f} \subset N \cdot \widetilde{Y}$ and $\|f\|_{L^{2}(\mathbb{R})} \leq\|u\|_{\ell_{N}^{2}}$, so by (5.24)

$$
\|f\|_{L^{2}(\tilde{X})} \leq C_{0} N^{-\beta_{0}}\|u\|_{\ell_{N}^{2}} .
$$

On the other hand, for all $j \in \mathbb{Z}_{N}$, we have for all $j \in X$

$$
\frac{1}{\sqrt{N}} f\left(\frac{j}{N}\right)=\mathcal{F}_{N}^{*} \mathbb{1}_{Y} u(j) \cdot\left(\mathcal{F}^{-1} \chi\right)\left(\frac{j}{N}\right) .
$$

Consider the nonoverlapping collection of intervals

$$
I_{j}:=\left[\frac{j}{N}-\frac{1}{2 N}, \frac{j}{N}+\frac{1}{2 N}\right] \subset \tilde{X}, \quad j \in X .
$$

Using that $\left(|f|^{2}\right)^{\prime}=2 \operatorname{Re}\left(\bar{f} f^{\prime}\right)$, we have

$$
\left|\mathcal{F}_{N}^{*} \mathbb{1}_{Y} u(j)\right|^{2} \leq \frac{1}{c^{2} N}\left|f\left(\frac{j}{N}\right)\right|^{2} \leq C \int_{I_{j}}|f(x)|^{2} d x+\frac{C}{N} \int_{I_{j}}|f(x)| \cdot\left|f^{\prime}(x)\right| d x
$$

where $C$ denotes some constant depending only on $\delta, C_{R}, \chi$. Summing over $j \in X$ and using the Cauchy-Schwarz inequality, we obtain

$$
\left\|\mathbb{1}_{X} \mathcal{F}_{N}^{*} \mathbb{1}_{Y} u\right\|_{\ell_{N}^{2}}^{2} \leq C\|f\|_{L^{2}(\widetilde{X})}^{2}+\frac{C}{N}\|f\|_{L^{2}(\widetilde{X})} \cdot\left\|f^{\prime}\right\|_{L^{2}(\mathbb{R})} .
$$

Since $\operatorname{supp} \hat{f} \subset[-N, N]$, we have $\left\|f^{\prime}\right\|_{L^{2}(\mathbb{R})} \leq 10 N\|f\|_{L^{2}(\mathbb{R})} \leq 10 N\|u\|_{\ell_{N}^{2}}$ and thus by $(5.25)$

$$
\left\|\mathbb{1}_{X} \mathcal{F}_{N}^{*} \mathbb{1}_{Y} u\right\|_{\ell_{N}^{2}}^{2} \leq C N^{-\beta_{0}}\|u\|_{\ell_{N}^{2}}^{2}
$$

which gives (5.23) with $\beta=\beta_{0} / 2$. 
Combining Propositions 5.5 and 5.8, we obtain (5.8) for $\frac{1}{2} \leq \delta<1$, finishing the proof of Theorem 4.

Acknowledgements. We would like to thank Jean Bourgain for inspiring discussions on the fractal uncertainty principle, and Maciej Zworski for many helpful comments. We would also like to thank an anonymous referee for many suggestions to improve the manuscript. This research was conducted during the period SD served as a Clay Research Fellow.

\section{REFERENCES}

[Bo16] David Borthwick, Spectral theory of infinite-area hyperbolic surfaces, second edition, Birkhäuser, 2016.

[BoDy16] Jean Bourgain and Semyon Dyatlov, Spectral gaps without the pressure condition, to appear in Ann. Math. (2), arXiv:1612.09040.

[Do98] Dmitry Dolgopyat, On decay of correlations in Anosov flows, Ann. Math. (2) 147(1998), 357-390.

[DyJi17] Semyon Dyatlov and Long Jin, Resonances for open quantum maps and a fractal uncertainty principle, Comm. Math. Phys. 354(2017), 269-316.

[DyZa16] Semyon Dyatlov and Joshua Zahl, Spectral gaps, additive energy, and a fractal uncertainty principle, Geom. Funct. Anal. 26(2016), 1011-1094.

[GaRi89] Pierre Gaspard and Stuart Rice, Scattering from a classically chaotic repeller, J. Chem. Phys. 90(1989), 2225-2241.

[Ik88] Mitsuru Ikawa, Decay of solutions of the wave equation in the exterior of several convex bodies, Ann. Inst. Fourier 38(1988), 113-146.

[MOW17] Michael Magee, Hee Oh, and Dale Winter, Uniform congruence counting for Schottky semigroups in $\mathrm{SL}_{2}(\mathbb{Z})$, with an appendix by Jean Bourgain, Alex Kontorovich, and Michael Magee, J. für die reine und angewandte Mathematik, published online.

[Na05] Frédéric Naud, Expanding maps on Cantor sets and analytic continuation of zeta functions, Ann. de l'ENS (4) 38(2005), 116-153.

[No11] Stéphane Nonnenmacher, Spectral problems in open quantum chaos, Nonlinearity 24(2011), R123.

[NoZw09] Stéphane Nonnenmacher and Maciej Zworski, Quantum decay rates in chaotic scattering, Acta Math. 203(2009), 149-233.

[OhWi16] Hee Oh and Dale Winter, Uniform exponential mixing and resonance free regions for convex cocompact congruence subgroups of $\mathrm{SL}_{2}(\mathbb{Z})$, J. Amer. Math. Soc. 29(2016), 1069-1115.

[Pa76] Samuel James Patterson, The limit set of a Fuchsian group, Acta Math. 136(1976), 241-273.

[PeSt10] Vesselin Petkov and Luchezar Stoyanov, Analytic continuation of the resolvent of the Laplacian and the dynamical zeta function, Anal. PDE 3(2010), 427-489.

[St11] Luchezar Stoyanov, Spectra of Ruelle transfer operators for axiom A flows, Nonliearity 24(2011), 1089-1120.

[St12] Luchezar Stoyanov, Non-integrability of open billiard flows and Dolgopyat-type estimates, Erg. Theory Dyn. Syst. 32(2012), 295-313.

[Su79] Dennis Sullivan, The density at infinity of a discrete group of hyperbolic motions, Publ. Math. de l'IHES 50(1979), 171-202. 
E-mail address: dyatlov@math.mit.edu

Department of Mathematics, Massachusetts Institute of Technology, 77 Massachusetts Ave, Cambridge, MA 02139

E-mail address: long249@purdue.edu

Department of Mathematics, Purdue University, 150 N. University St, West Lafayette, IL 47907 